\title{
Conformation of the Galactose Ring Adopted in Solution and in Crystalline Form as Determined by Experimental and DFT ${ }^{1} \mathrm{H}$ NMR and Single-Crystal X-Ray Analysis
}

\author{
Mattias U. Roslund, Karel D. Klika, Reko L. Lehtilä, \\ Petri Tähtinen, Reijo Sillanpää, and Reko Leino
}

\section{Supporting Information}

Table of Contents:

General experimental details, NMR, X-ray, etc ................................................... 3

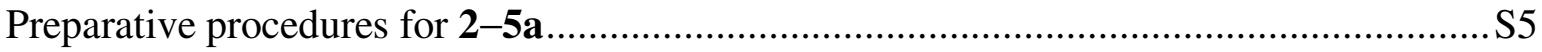

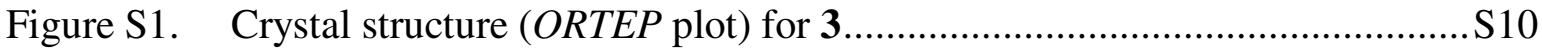

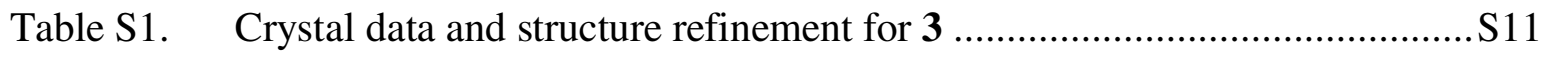

Table S2. Atomic coordinates and equivalent isotropic

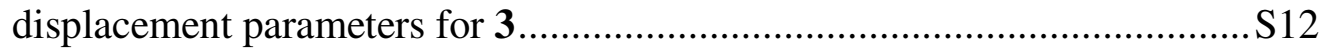

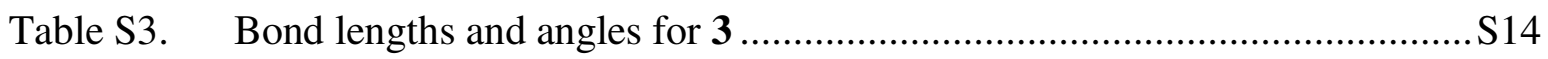

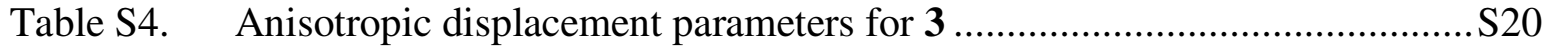

Table S5. Hydrogen coordinates and isotropic

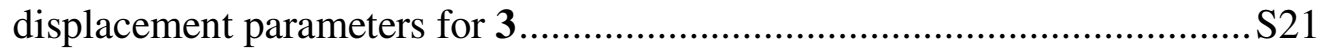

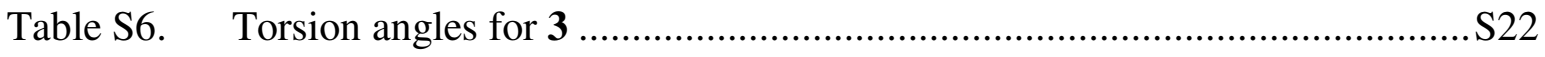

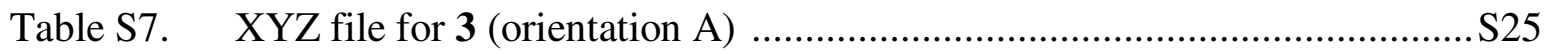


Table S8. XYZ file for 3 (orientation B) …........................................................ S27

Figure S2. Crystal structure (ORTEP plot) for 5a............................................. 29

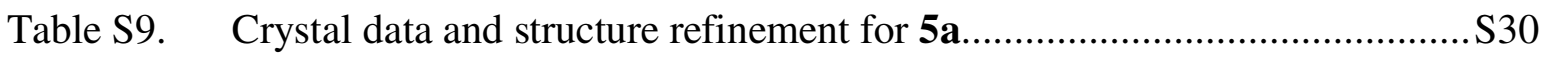

Table S10. Atomic coordinates and equivalent isotropic

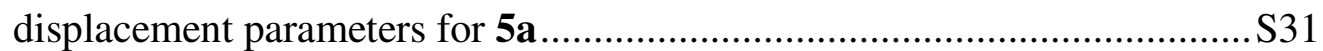

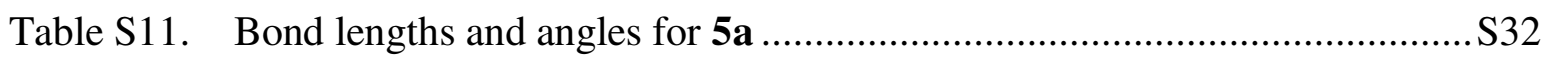

Table S12. Anisotropic displacement parameters for 5a ....................................S35

Table S13. Hydrogen coordinates and isotropic

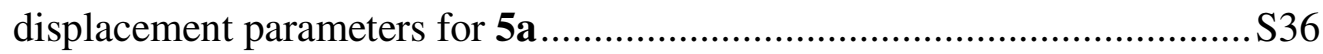

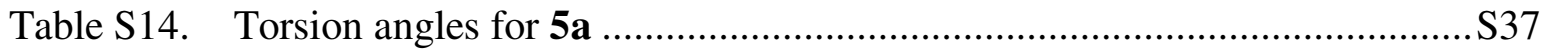

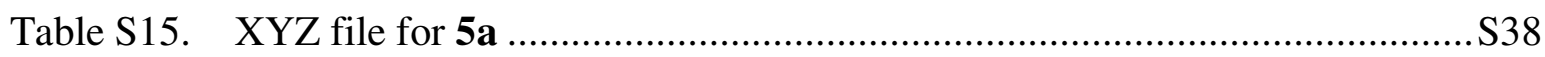

Table S16. Cartesian coordinates from DFT calculations for $1 \mathbf{1 a}$ (ex 5a) .....................S39

Table S17. Cartesian coordinates from DFT calculations for 19 (ex 3)......................S40

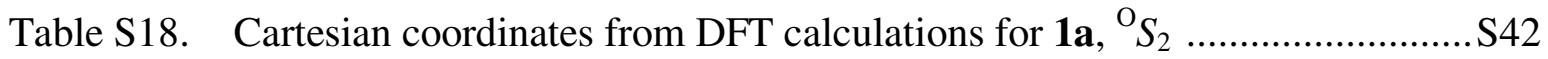

Table S19. Cartesian coordinates from DFT calculations for 3, unopt. ........................S43

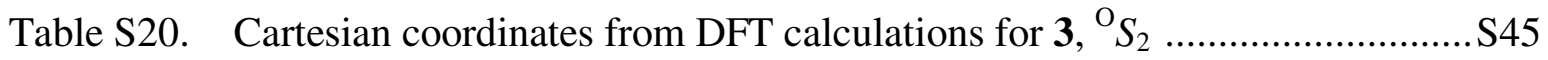

Table S21. Cartesian coordinates from DFT calculations for 5a, unopt. .....................S47

Table S22. Cartesian coordinates from DFT calculations for 5a ...............................S49 
General. Melting points were determined using Stuart Scientific melting point SMP1 apparatus and are uncorrected. TLC was performed using silica gel $\mathrm{F}_{254}$ precoated aluminum sheets and visualized by charring with $25 \% \mathrm{H}_{2} \mathrm{SO}_{4}$ in methanol. Column chromatography was performed using silica gel 60 enriched with $0.1 \%$ Ca to minimize hydrolysis of acid-labile protecting groups. All operations with air- or moisture-sensitive reagents were conducted in an inert atmosphere using standard Schlenk and vacuum techniques. Solvents were dried and distilled under argon prior to use when applicable or purchased as anhydrous from commercial sources. 2- $N$-Phthalimido-3,4,6-tri- $O$-acetyl-2-deoxy- $\alpha$-D-bromoglucose, $\alpha$ D-galactose (1a), and 1,2,3,4,6-pentaacetyl- $\beta$-D-galactopyranose (5b) were obtained commercially and were used as supplied. Optical rotations are expressed as $[\alpha]_{\mathrm{D}}$ values in deg $\mathrm{mL} \mathrm{dm}^{-1} \mathrm{~g}^{-1}$ units and were measured using a cell of volume $1 \mathrm{~mL}$ and length $10 \mathrm{~cm}$.

NMR spectra were acquired at $11.75 \mathrm{~T}$ using a spectrometer equipped with either a $5 \mathrm{~mm}$ normal configuration tunable probe or a $5 \mathrm{~mm}$ inverse z-axis fg probe operating at $500.16 \mathrm{MHz}$ for ${ }^{1} \mathrm{H}$ and 125.77 $\mathrm{MHz}$ for ${ }^{13} \mathrm{C}$. 1-D ${ }^{1} \mathrm{H}$ spectra were acquired with single-pulse excitation, $45^{\circ}$ flip angle, pulse recycle time of $9.5 \mathrm{~s}$ and with spectral widths of $7 \mathrm{kHz}$ consisting of $64 \mathrm{k}$ data points (digital resolution $0.11 \mathrm{~Hz} / \mathrm{pt}$ ), zero-filled to $128 \mathrm{k}$ prior to Fourier transformation. 1-D ${ }^{1} \mathrm{H}$ spectra were processed with a double exponential to effect resolution enhancement prior to spin analysis which was performed using Perch $^{1}$ iteration software for the extraction of $\delta_{\mathrm{H}}$ and $J_{\mathrm{H}, \mathrm{H}}$. Since the reliable extraction of small couplings approaching the linewidth is heavily dependent on whether they are to a degree resolvable on at least one spin for Perch to reliably extract them, only those couplings reliably extracted by Perch are reported whilst couplings buried in the linewidth on both interacting spins are not reported (i.e., not extracted) even if their likely presence is probable or is evident from homodecoupling experiments. For 1-D NOE difference experiments, the intensity of enhancement was integrated relative to the intensity of the irradiated signal set to $-100 \%$. FG DQF COSY, TOCSY, and NOESY spectra were all acquired in phasesensitive mode and processed with zero-filling $(\times 2, \times 4)$ and exponential weighting $(1-3 \mathrm{~Hz})$ applied in 
both dimensions prior to Fourier transformation. 1-D ${ }^{13} \mathrm{C}$ spectra were acquired with single-pulse excitation, $45^{\circ}$ flip angle, pulse recycle time of $3.5 \mathrm{~s}$ and with spectral widths of $30 \mathrm{kHz}$ consisting of 64 $\mathrm{k}$ data points (digital resolution $0.46 \mathrm{~Hz} / \mathrm{pt}$ ), zero-filled to $128 \mathrm{k}$ and with $1 \mathrm{~Hz}$ exponential weighting generally applied prior to Fourier transformation. DEPT $135^{\circ}$ were acquired under similar conditions but with a post acquisition delay time of $3 \mathrm{~s}$. FG HMQC and FG HMBC experiments were both acquired in magnitude mode and processed with zero-filling $(\times 2, \times 4)$, a $2 \pi / 3$-shifted sinebell function, and exponential weighting $(3-5,10-20 \mathrm{~Hz})$ applied in both dimensions prior to Fourier transformation. Both HMQC and HMBC spectra utilized a ${ }^{1} J_{\mathrm{H}, \mathrm{C}}$ coupling of $180 \mathrm{~Hz}$, whilst the HMBC correlations were optimized for a long-range ${ }^{\mathrm{n}} J_{\mathrm{H}, \mathrm{C}}$ coupling of $8 \mathrm{~Hz}$. For all 2-D spectra, the spectral widths and resolution were appropriately optimized from the 1-D spectra.

X-Ray crystallographic data were collected at $173 \mathrm{~K}$ on an area-detector diffractometer using graphite-monochromatized Mo- $\mathrm{K}_{\alpha}$ radiation $(\lambda=0.71073 \AA)$. Lattice parameters were determined from 10 images recorded with $1^{\circ} \varphi$ scans and subsequently refined on all data. Data collections were performed using $\varphi$ and $\omega$ scans with $2^{\circ}$ steps. The crystal-to-detector distance was $35 \mathrm{~mm}$ for $\mathbf{3}$ and 30 mm for 5a. The data were processed using DENZO-SMN v0.93.0. ${ }^{2}$ The structures were solved by direct methods using the SIR92 program ${ }^{3}$ and full-matrix least-squares refinements on $F^{2}$ were performed using the SHELX-97 program. ${ }^{4}$ All heavy atoms were refined anisotropically except for the disordered atoms in 3 . The methyl and phenyl hydrogen atoms were included at calculated distances from their host atoms with fixed displacement parameters. The rest of the hydrogen atoms were refined isotropically or with fixed displacement parameters. Figures were drawn using ORTEP-3 for Windows. ${ }^{5}$

Electron impact high-resolution mass spectra (EIMS) were acquired using a direct insert probe scanning from 50 to $1500 \mathrm{amu}$ and using electrons energized to $70 \mathrm{eV}$. Accurate mass measurements were performed using a peak matching technique with PFK as a reference substance at a resolution of $8,000-10,000$ (at 10\% peak height). 
Computational Method. Structures were optimized with the Gaussian $98 W$ program package ${ }^{6}$ using DFT at the B3LYP/6-31G(d,p) level of theory. ${ }^{7,8,9,10,11}$ This level of theory for geometry optimization was previously found to be adequate for the reliable calculation of ${ }^{1} \mathrm{H}$ NMR parameters. ${ }^{12,13}$ For the evaluation of the spin-spin coupling constants, the Fermi contact contribution was calculated using Gaussian $98 \mathrm{~W}$ by finite perturbation theory (Field keyword) at the spin-unrestricted B3LYP/cc$\mathrm{pVTZ}^{14}$ level. Tight SCF convergence criteria were always used. ${ }^{13,15}$ A perturbation of $10^{-2}$ au was applied to a selected proton (H-1, H-2, or H-4) in order to obtain all couplings to this proton. As previously discussed, ${ }^{12,13}$ the calculations of the paramagnetic and diamagnetic spin-orbit terms (PSO and DSO, respectively) were omitted since their addition in the general case leads to a negligible effect because they are of similar magnitude and opposite in sign, at least for the case of homonuclear proton couplings. ${ }^{12,13,16,17,18,19}$ The spin-dipole (SD) term was not calculated as it is generally known to be negligible and is computationally expensive ${ }^{20}$ Calibration of the calculated coupling constants was then made as described previously ${ }^{12}$ by adjusting their value using the equation: $J_{\text {calib }}=1.1169 \times J_{\text {uncalib }}-$ 0.0277 .

1,2:3,4-Di- $O$-isopropylidene- $\alpha$-D-galactopyranose (2) was prepared according to literature ${ }^{21}$ from D-galactose (1). Anhydrous D-galactose $(38.15 \mathrm{~g}, 211.8 \mathrm{mmol})$ together with anhydrous $\mathrm{CuSO}_{4}(80.61 \mathrm{~g}$, $505.0 \mathrm{mmol})$ and concentrated $\mathrm{H}_{2} \mathrm{SO}_{4}(4.1 \mathrm{~mL})$ was added to $800 \mathrm{~mL}$ of dry acetone and stirred for $24 \mathrm{~h}$ under argon at room temperature. Following this, the $\mathrm{CuSO}_{4}$ was removed by filtration and washed with dry acetone. $\mathrm{CaOH}$ was added portion-wise to the combined filtrates until the solution was neutralized. The unreacted $\mathrm{CaOH}$ and $\mathrm{CaSO}_{4}$ were then filtered off and washed with acetone. The combined filtrates were then concentrated in vacuo to a yellow oil which was distilled at $169-170{ }^{\circ} \mathrm{C}$ (10 mbar) to provide the desired product 2 in $95 \%$ yield. $[\alpha]_{\mathrm{D}}^{25}\left(c 10 \mathrm{~g} \mathrm{~L}^{-1}\right.$ in $\left.\mathrm{CHCl}_{3}\right)$ : -58.9. EIMS: calcd for $\mathrm{C}_{12} \mathrm{H}_{20} \mathrm{O}_{6}[\mathrm{M}-$ 
$\left.\mathrm{CH}_{3}\right]^{+}$245.1025; found 245.1029. $\mathrm{C}_{12} \mathrm{H}_{20} \mathrm{O}_{6}$ (260.3): calcd. C 55.37, H 7.74, O 36.88; found C 55.5, H 7.8. ${ }^{1} \mathrm{H}$ and ${ }^{13} \mathrm{C}$ NMR parameters are listed in Tables $1-3$ and are in accord with literature values. ${ }^{22,23}$

\section{1,2:3,4-Di- $O$-isopropylidene-6- $O$-(2- $N$-phthalimido-3,4,6-tri- $O$-acetyl-2-deoxy- $\alpha$-D-}

glucopyranosyl)- $\alpha$-D-galactopyranose (3) was prepared by the reaction of 2 (1.00 g, $3.84 \mathrm{mmol})$ with 2$N$-phthalimido-3,4,6-tri- $O$-acetyl-2-deoxy- $\alpha$-D-bromoglucose (1.91 g, $3.84 \mathrm{mmol})$ in dichloromethane (50 $\mathrm{mL})$ and toluene $(30 \mathrm{~mL})$ in the presence of $4 \AA$ molecular sieves $(2.0 \mathrm{~g})$ together with $2,2,6,6$ tetramethylpiperidine $(0.3 \mathrm{~mL})$ and $\operatorname{AgOTf}(2.60 \mathrm{~g})$ at $-50{ }^{\circ} \mathrm{C}$. Over the course of the reaction $(4 \mathrm{~h} 40$ $\min$ ), the temperature was allowed to rise to $0{ }^{\circ} \mathrm{C}$. The reaction mixture was filtered through a plug of Celite and dried with $\mathrm{Na}_{2} \mathrm{SO}_{4}$. Column chromatography with gradient elution of toluene/ethyl acetate gave disaccharide 3 in $70 \%$ yield as white crystals, mp $271-272{ }^{\circ} \mathrm{C}$. Crystals-long needles consisting of oblate hexagons in the transverse plane-amenable to X-ray analysis were grown by slow evaporation from a mixed solvent system of methanol and chloroform which was left to stand till dry. See Table S1 for crystal data and structure refinement details. Full structural information has also been deposited with the Cambridge Crystallographic Data Centre. $[\alpha]_{\mathrm{D}}^{25}$ (c $10 \mathrm{~g} \mathrm{~L}^{-1}$ in $\left.\mathrm{CHCl}_{3}\right)$ : -23.0 . EIMS: calcd for $\mathrm{C}_{32} \mathrm{H}_{39} \mathrm{NO}_{15}[\mathrm{M}]^{+}$677.2320; found 677.2326. Anal. Calc for $\mathrm{C}_{32} \mathrm{H}_{39} \mathrm{NO}_{15}$ : C 56.72, H 5.80, N 2.07, O 35.42. Found: C 56.68, H 5.81, N 2.04. ${ }^{1} \mathrm{H}$ and ${ }^{13} \mathrm{C}$ NMR parameters are listed in Tables $1-3$ and are in accord with literature values. $^{24}$

1,2:3,4-Di- $O$-isopropylidene-6- $O$-tert-butyldiphenylsilyl- $\alpha$-D-galactopyranose (4). To a mixture of $2(6.82 \mathrm{~g}, 26.18 \mathrm{mmol})$ and $\mathrm{DBU}(6.8 \mathrm{~mL}, 52.36 \mathrm{mmol})$ in $\mathrm{CH}_{2} \mathrm{Cl}_{2}(5.0 \mathrm{~mL})$ at $0{ }^{\circ} \mathrm{C}$ under stirring was added tert-butyldiphenylchlorosilane. The temperature was allowed to rise to room temperature and after $17 \mathrm{~h}$ the reaction mixture was washed sequentially with aqueous $\mathrm{Na}_{2} \mathrm{CO}_{3}$ (sat, $3 \times 50 \mathrm{~mL}$ ) and aqueous brine (sat, $1 \times 50 \mathrm{~mL}$ ). The solution was dried over $\mathrm{Na}_{2} \mathrm{SO}_{4}$, filtered and the solvent removed in vacuo to provide 4 as a yellow oil in $82 \%$ yield (10.64 g). EIMS: calcd for $\mathrm{C}_{27} \mathrm{H}_{35} \mathrm{O}_{6} \mathrm{Si}\left[\mathrm{M}-\mathrm{CH}_{3}\right]^{+} 483.2203$; 
found 483.2209. $\mathrm{C}_{28} \mathrm{H}_{38} \mathrm{O}_{6} \mathrm{Si}$ (498.7): calcd. C 67.44, H 7.68, O 19.25, Si 5.63; found C 67.3, H 7.6. ${ }^{1} \mathrm{H}$ and ${ }^{13} \mathrm{C}$ NMR parameters are listed in Tables $1-3$ and are in accord with literature values. ${ }^{25}$

1,2,3,4,6-Pentaacetyl- $\alpha$-D-galactopyranose (5a) was prepared from 1 (10.01 g, $55.56 \mathrm{mmol})$ in acetic anhydride $(60 \mathrm{~mL})$ together with pyridine $(60 \mathrm{~mL})$ and 1,4-dioxane $(60 \mathrm{~mL})$. The mixture was stirred for $66 \mathrm{~h}$ at ambient temperature and then cooled on an ice-bath prior to the addition of methanol. The solvent was removed and the residue taken up $\mathrm{CH}_{2} \mathrm{Cl}_{2}$ and extracted with water ( $2 \times$ equal volume). The organic phase was dried over $\mathrm{Na}_{2} \mathrm{SO}_{4}$, filtered and the solvent once again removed in vacuo. The majority of the $\beta$ anomer (5b) was first removed from the mixture of $\alpha$ and $\beta$ anomers (5a and $\mathbf{5 b}$ ) by crystallization from ethanol. Pure 5a was then obtained from the remaining mixture by repeated recrystallization from diethyl ether/petroleum ether. Yellow, plate-like crystals amenable to X-ray analysis were grown by slow evaporation from a mixed solvent system of methanol and chloroform that was left to stand till dry. See Table S9 for crystal data and structure refinement details. Full structural information has also been deposited with the Cambridge Crystallographic Data Centre. $[\alpha]_{\mathrm{D}}^{25}\left(c 10 \mathrm{~g} \mathrm{~L}^{-1}\right.$ in $\mathrm{CHCl}_{3}$ ): +102.3. EIMS: calcd for $\mathrm{C}_{14} \mathrm{H}_{19} \mathrm{O}_{9}[\mathrm{M}-\mathrm{OAc}]^{+} 331.1029$; found 331.1030. $\mathrm{C}_{16} \mathrm{H}_{22} \mathrm{O}_{11}(390.3)$ : calcd. C 49.23, H 5.68, O 45.09; found C 49.4, H 5.7. ${ }^{1} \mathrm{H}$ and ${ }^{13} \mathrm{C}$ NMR parameters are listed in Tables 1-3 and are in accord with literature values.

\section{References}

(1) see for example, Laatikainen, R.; Niemitz, M.; Weber, U.; Sundelin, J.; Hassinen, T.; Vepsäläinen, J. J. Magn. Reson., Ser. A 1996, 120, 1, or the program website at http://www.uku.fi/perch.html.

(2) Otwinowski, Z.; Minor, W. In Methods in Enzymology, Macromolecular Crystallography, Part A; Carter, C. W. Jr.; Sweet, R. M., Eds.; Academic Press: New York, 1997, pp. 307-326. 
Altomare, A.; Cascarano, G.; Giacovazzo, C.; Gualiardi, A.; Burla, M. C.; Polidori, G.;

Camalli, M.. J. Appl. Cryst. 1994, 27, 435.

(4) Sheldrick, G. M. SHELX-97, University of Göttingen, Germany, 1997.

Farrugia, L. J. J. Appl. Cryst. 1997, 30, 565.

Frisch, M. J.; Trucks, G. W.; Schlegel, H. B.; Scuseria, G. E.; Robb, M. A.; Cheeseman, J. R.; Zakrzewski, V. G.; Montgomery, J. A., Jr.; Stratmann, R. E.; Burant, J. C.; Dapprich, S.; Millam, J. M.; Daniels, A. D.; Kudin, K. N.; Strain, M. C.; Farkas, O.; Tomasi, J.; Barone, V.; Cossi, M.; Cammi, R.; Mennucci, B.; Pomelli, C.; Adamo, C.; Clifford, S.; Ochterski, J.; Petersson, G. A.; Ayala, P. Y.; Cui, Q.; Morokuma, K.; Malick, D. K.; Rabuck, A. D.; Raghavachari, K.; Foresman, J. B.; Cioslowski, J.; Ortiz, J. V.; Stefanov, B. B.; Liu, G.; Liashenko, A.; Piskorz, P.; Komaromi, I.; Gomperts, R.; Martin, R. L.; Fox, D. J.; Keith, T.; Al-Laham, M. A.; Peng, C. Y.; Nanayakkara, A.; Gonzalez, C.; Challacombe, M.; Gill, P. M. W.; Johnson, B. G.; Chen, W.; Wong, M. W.; Andres, J. L.; Head-Gordon, M.; Replogle, E. S.; Pople, J. A. Gaussian 98, revision A.11; Gaussian, Inc.: Pittsburgh, PA, 2001.

Becke, A. D. Phys. Rev. A 1988, 38, 3098-3100.

Becke, A. D. J. Chem. Phys. 1993, 98, 5648-5652.

Lee, C.; Yang, W.; Parr, R. G. Phys. Rev. B 1988, 37, 785-789.

Miehlich, B.; Savin, A.; Stoll, H.; Preuss, H. Chem. Phys. Lett. 1989, 157, 200-206.

Hehre, W. J.; Radom, L.; Schleyer, P. v. R.; Pople, J. A. In Ab Initio Molecular Orbital Theory; Wiley: New York, NY, 1986.

(12) Tähtinen, P.; Bagno, A.; Klika, K. D.; Pihlaja, K. J. Am. Chem. Soc. 2003, 125, 4609-4618 and references cited therein. Bagno, A. Chem. Eur. J. 2001, 7, 1652-1661.

Kendall, R. A.; Dunning, T. H., Jr.; Harrison, R. J. J. Chem. Phys. 1992, 96, 6796-6806. 
(15) Bagno, A. Chem. Eur. J. 2000, 6, 2925-2930.

(16) Lazzeretti, P.; Malagoli, M.; Zanasi, R.; Della, E. W.; Lochert, I. J.; Giribet, C. G.; Ruiz de Azúa, M. C.; Contreras, R. H. J. Chem. Soc., Faraday Trans. 1995, 91, 4031-4035.

(17) Onak, T.; Jaballas, J.; Barfield, M. J. Am. Chem. Soc. 1999, 121, 2850-2856.

(18) Czernek, J.; Lang, J.; Sklenár, V. J. Phys. Chem. A 2000, 104, 2788-2792.

(19) Sychrovský, V.; Gräfenstein, J.; Cremer, D. J. Chem. Phys. 2000, 113, 3530-3547.

(20) Helgaker, T.; Jaszunski, M.; Ruud, K. Chem. Rev. 1999, 99, 293-352.

(21) Schmidt, O. T. Methods Carbohyd. Chem. 2, 1963, 318-325.

(22) Rauter, A. P.; Ramôa-Ribeiro, F.; Fernandes, A. C.; Figueiredo, J. A. Tetrahedron 1995, 51, 6529-6540 and references cited therein.

(23) Asakura, J.; Matsubara, Y.; Yoshihara, M. J. Carbohyd. Chem. 1996, 15, 231-239 and references cited therein.

(24) Cao, S.; Hernández-Matéo, F.; Roy, R. J. Carbohyd. Chem. 1998, 17, 609-631.

(25) Nashed, E. M.; Glaudemans, C. P. J. J. Org. Chem. 1987, 52, 5255-5260. 


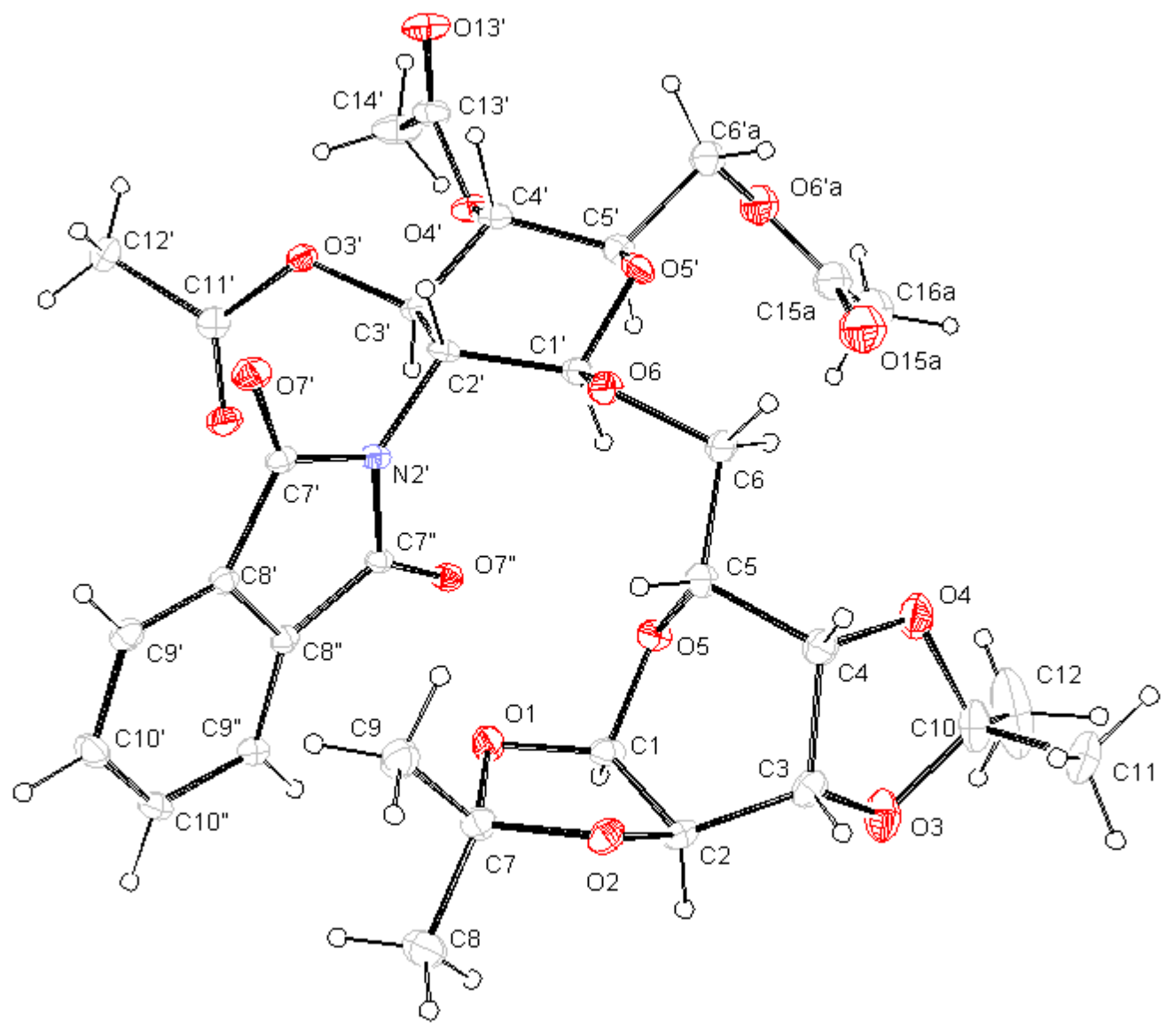

The structure of $\mathbf{3}$ derived from single-crystal X-ray analysis (ORTEP plot). The thermal displacement parameters are drawn at $20 \%$ probability. From the one crystal, two orientations, A and B, were obtained for the $\mathrm{C}_{6}$-acetyl fragment (orientation $\mathrm{A}$ is shown). 
Table S1. Crystal data and structure refinement for $\mathbf{3}$.

Empirical formula

Formula weight

Temperature

Wavelength

Crystal system

Space group

Unit cell dimensions

Volume

Z

Density (calculated)

Absorption coefficient

$\mathrm{F}(000)$

Crystal size

Theta range for data collection

Index ranges

Reflections collected

Independent reflections

Completeness to theta $=25.03^{\circ}$

Absorption correction

Refinement method

Data / restraints / parameters

Goodness-of-fit on $\mathrm{F}^{2}$

Final $R$ indices $[I>2 \sigma(I)]$

$\mathrm{R}$ indices (all data)

Absolute structure parameter

Largest diff. peak and hole
$\mathrm{C}_{32} \mathrm{H}_{39} \mathrm{NO}_{15}$
677.64
173 (2) K
$0.71073 \AA$

hexagonal

P65

$a=23.8464$ (6) $\AA \quad \alpha=90^{\circ}$

$b=23.8464$ (6) $\AA \quad \beta=90^{\circ}$

$c=10.1843(2) \AA \quad \gamma=120^{\circ}$

$5,015.4(2) \AA^{3}$

6

$1.346 \mathrm{Mg} / \mathrm{m}^{3}$

$0.108 \mathrm{~mm}^{-1}$

2,148

$0.30 \times 0.22 \times 0.12 \mathrm{~mm}$

$2.23^{\circ}-25.03^{\circ}$

$-28 \leq \mathrm{h} \leq 28,-24 \leq \mathrm{k} \leq 23,-11 \leq 1 \leq 11$

10,570

$5,722[\mathrm{R}(\mathrm{int})=0.0325]$

$99.5 \%$

none

full-matrix least-squares on $\mathrm{F}^{2}$

5,722 / 1 / 459

1.024

$\mathrm{R} 1=0.0540, \mathrm{wR} 2=0.1214$

$\mathrm{R} 1=0.0758, \mathrm{wR} 2=0.1335$

$0.1(11)$

0.494 and -0.383 e. $\AA^{-3}$ 
Table S2. Atomic coordinates $\left(\times 10^{4}\right)$ and equivalent isotropic displacement parameters $\left(A^{2} \times 10^{3}\right)$ for 3 .

$\mathrm{U}(\mathrm{eq})$ is defined as one third of the trace of the orthogonalized Uij tensor.

\begin{tabular}{|c|c|c|c|c|}
\hline & $\mathrm{x}$ & $\mathrm{Y}$ & $z$ & $\mathrm{U}(\mathrm{eq})$ \\
\hline$O\left(3^{\prime}\right)$ & $810(1)$ & $4760(1)$ & $-3479(2)$ & $30(1)$ \\
\hline$O\left(4^{\prime}\right)$ & $-46(1)$ & $3455(1)$ & $-4181(2)$ & $39(1)$ \\
\hline$O\left(5^{\prime}\right)$ & $1387(1)$ & $3456(1)$ & $-2937(2)$ & $40(1)$ \\
\hline$O\left(7^{\prime}\right)$ & $2352(1)$ & $5796(1)$ & $-1834(2)$ & $37(1)$ \\
\hline $\mathrm{O}(7 ")$ & $655(1)$ & $4089(1)$ & $256(2)$ & $28(1)$ \\
\hline$O\left(11^{\prime}\right)$ & $311(1)$ & $4950(1)$ & $-1791(3)$ & $42(1)$ \\
\hline$O\left(13^{\prime}\right)$ & $180(1)$ & $3737(2)$ & $-6301(3)$ & $50(1)$ \\
\hline$O(1)$ & $2062(1)$ & $4698(1)$ & $2252(2)$ & $37(1)$ \\
\hline$O(2)$ & $2850(1)$ & $4629(1)$ & $3331(2)$ & $34(1)$ \\
\hline$O(3)$ & $2011(1)$ & $2887(1)$ & $3334(3)$ & $54(1)$ \\
\hline$O(4)$ & $2248(2)$ & $2801(2)$ & $1249(3)$ & $64(1)$ \\
\hline$O(5)$ & $1776(1)$ & $3737(1)$ & $1196(2)$ & $31(1)$ \\
\hline$O(6)$ & $2158(1)$ & $4089(1)$ & $-1463(2)$ & $38(1)$ \\
\hline $\mathrm{N}\left(2^{\prime}\right)$ & $1513(1)$ & $4836(1)$ & $-1015(3)$ & $25(1)$ \\
\hline$C\left(1^{\prime}\right)$ & $1515(2)$ & $3852(2)$ & $-1803(3)$ & $33(1)$ \\
\hline$C\left(2^{\prime}\right)$ & $1435(2)$ & $4431(2)$ & $-2155(3)$ & $26(1)$ \\
\hline$C\left(3^{\prime}\right)$ & $784(2)$ & $4215(2)$ & $-2814(3)$ & $26(1)$ \\
\hline$C\left(4^{\prime}\right)$ & $626(2)$ & $3704(2)$ & $-3864(3)$ & $32(1)$ \\
\hline$C\left(5^{\prime}\right)$ & $726(2)$ & $3163(2)$ & $-3344(4)$ & $39(1)$ \\
\hline$C\left(7^{\prime}\right)$ & $1960(2)$ & $5496(2)$ & $-976(3)$ & $25(1)$ \\
\hline$C(7 ")$ & $1098(2)$ & $4638(2)$ & $71(3)$ & $23(1)$ \\
\hline$C\left(8^{\prime}\right)$ & $1837(2)$ & $5740(2)$ & $267(3)$ & $25(1)$ \\
\hline$C(8 ")$ & $1311(2)$ & $5222(2)$ & $895(3)$ & $25(1)$ \\
\hline$C\left(9^{\prime}\right)$ & $2150(2)$ & $6347(2)$ & $809(3)$ & $32(1)$ \\
\hline$C(9 ")$ & $1088(2)$ & $5298(2)$ & $2091(3)$ & $30(1)$ \\
\hline$C\left(10^{\prime}\right)$ & $1920(2)$ & $6434(2)$ & $2002(4)$ & $38(1)$ \\
\hline C (10") & $1397(2)$ & $5921(2)$ & $2623(3)$ & $35(1)$ \\
\hline$C\left(11^{\prime}\right)$ & $541(2)$ & $5081(2)$ & $-2879(4)$ & $33(1)$ \\
\hline$C\left(12^{\prime}\right)$ & $570(2)$ & $5597(2)$ & $-3755(4)$ & $37(1)$ \\
\hline$C\left(13^{\prime}\right)$ & $-202(2)$ & $3540(2)$ & $-5414(4)$ & $38(1)$ \\
\hline$C\left(14^{\prime}\right)$ & $-894(2)$ & $3360(3)$ & $-5484(4)$ & $56(1)$ \\
\hline$C(1)$ & $1819(2)$ & $4022(2)$ & $2424(3)$ & $32(1)$ \\
\hline$C(2)$ & $2298(2)$ & $3984(2)$ & $3373(4)$ & $32(1)$ \\
\hline$C(3)$ & $2518(2)$ & $3516(2)$ & $2995(4)$ & $35(1)$ \\
\hline$C(4)$ & $2629(2)$ & $3478(2)$ & $1524(4)$ & $41(1)$ \\
\hline$C(5)$ & $2388(2)$ & $3850(2)$ & $708(4)$ & $33(1)$ \\
\hline$C(6)$ & $2280(2)$ & $3647(2)$ & $-716(3)$ & $40(1)$ \\
\hline$C(7)$ & $2606(2)$ & $5056(2)$ & $3093(4)$ & $35(1)$ \\
\hline$C(8)$ & $2396(2)$ & $5229(2)$ & $4356(4)$ & $53(1)$ \\
\hline$C(9)$ & $3109(2)$ & $5642(2)$ & $2386(4)$ & $44(1)$ \\
\hline$C(10)$ & $2066(2)$ & $2459(2)$ & $2454(5)$ & $51(1)$ \\
\hline$C(11)$ & $2569(2)$ & $2300(2)$ & 2891 (5) & $55(1)$ \\
\hline$C(12)$ & $1405(3)$ & $1859(3)$ & $2320(10)$ & $117(3)$ \\
\hline$C\left(\sigma^{\prime} A\right)$ & $695(7)$ & $2675(6)$ & $-4343(12)$ & $42(4)$ \\
\hline$O\left(\sigma^{\prime} A\right)$ & $78(3)$ & $2071(3)$ & $-3947(7)$ & $53(2)$ \\
\hline$O(15 \mathrm{~A})$ & $549(4)$ & $1891(4)$ & $-2275(8)$ & $75(2)$ \\
\hline$C(15 A)$ & $62(5)$ & $1720(5)$ & $-2984(10)$ & $51(3)$ \\
\hline$C(16 \mathrm{~A})$ & $-556(6)$ & $1154(6)$ & $-2741(13)$ & $75(3)$ \\
\hline
\end{tabular}




$\begin{array}{lrrrr}C(6 ' B) & 542(6) & 2665(6) & -4444(11) & 51(4) \\ O(6 ' B) & 552(3) & 2103(3) & -3926(7) & 69(2) \\ O(15 B) & 135(4) & 1591(4) & -5826(9) & 100(3) \\ C(15 B) & 291(6) & 1563(6) & -4693(13) & 91(4) \\ C(16 B) & 254(9) & 977(8) & -4029(18) & 143(6)\end{array}$


Table S3. Bond lengths [A] and angles [deg] for 3.

\begin{tabular}{|c|c|}
\hline$O\left(3^{\prime}\right)-C\left(11^{\prime}\right)$ & $1.363(4)$ \\
\hline$O\left(3^{\prime}\right)-C\left(3^{\prime}\right)$ & $1.440(4)$ \\
\hline $\mathrm{O}\left(4^{\prime}\right)-\mathrm{C}\left(13^{\prime}\right)$ & $1.353(5)$ \\
\hline $\mathrm{O}\left(4^{\prime}\right)-\mathrm{C}\left(4^{\prime}\right)$ & $1.440(5)$ \\
\hline $\mathrm{O}\left(5^{\prime}\right)-\mathrm{C}\left(1^{\prime}\right)$ & $1.424(4)$ \\
\hline $\mathrm{O}\left(5^{\prime}\right)-\mathrm{C}\left(5^{\prime}\right)$ & $1.429(5)$ \\
\hline $\mathrm{O}\left(7^{\prime}\right)-\mathrm{C}\left(7^{\prime}\right)$ & $1.217(4)$ \\
\hline $\mathrm{O}(7 ")-\mathrm{C}(7 ")$ & $1.217(4)$ \\
\hline$O\left(11^{\prime}\right)-C\left(11^{\prime}\right)$ & $1.206(4)$ \\
\hline$O\left(13^{\prime}\right)-C\left(13^{\prime}\right)$ & $1.199(4)$ \\
\hline $\mathrm{O}(1)-\mathrm{C}(1)$ & $1.426(5)$ \\
\hline $\mathrm{O}(1)-\mathrm{C}(7)$ & $1.428(4)$ \\
\hline $\mathrm{O}(2)-\mathrm{C}(7)$ & $1.425(4)$ \\
\hline $\mathrm{O}(2)-\mathrm{C}(2)$ & $1.441(5)$ \\
\hline$O(3)-C(10)$ & $1.415(5)$ \\
\hline$O(3)-C(3)$ & $1.420(5)$ \\
\hline$O(4)-C(10)$ & $1.416(6)$ \\
\hline $\mathrm{O}(4)-\mathrm{C}(4)$ & $1.429(5)$ \\
\hline $\mathrm{O}(5)-\mathrm{C}(1)$ & $1.403(4)$ \\
\hline$O(5)-C(5)$ & $1.433(4)$ \\
\hline$O(6)-C\left(1^{\prime}\right)$ & $1.386(4)$ \\
\hline $\mathrm{O}(6)-\mathrm{C}(6)$ & $1.443(4)$ \\
\hline $\mathrm{N}\left(2^{\prime}\right)-\mathrm{C}\left(7^{\prime}\right)$ & $1.393(4)$ \\
\hline $\mathrm{N}\left(2^{\prime}\right)-\mathrm{C}\left(7^{\prime \prime}\right)$ & $1.401(4)$ \\
\hline $\mathrm{N}\left(2^{\prime}\right)-\mathrm{C}\left(2^{\prime}\right)$ & $1.461(4)$ \\
\hline$C\left(1^{\prime}\right)-C\left(2^{\prime}\right)$ & $1.527(5)$ \\
\hline $\mathrm{C}\left(1^{\prime}\right)-\mathrm{H}\left(1^{\prime}\right)$ & $1.02(4)$ \\
\hline$C\left(2^{\prime}\right)-C\left(3^{\prime}\right)$ & $1.525(5)$ \\
\hline $\mathrm{C}\left(2^{\prime}\right)-\mathrm{H}\left(2^{\prime}\right)$ & $1.01(4)$ \\
\hline$C\left(3^{\prime}\right)-C\left(4^{\prime}\right)$ & $1.520(5)$ \\
\hline $\mathrm{C}\left(3^{\prime}\right)-\mathrm{H}\left(3^{\prime}\right)$ & $0.94(4)$ \\
\hline$C\left(4^{\prime}\right)-C\left(5^{\prime}\right)$ & $1.520(6)$ \\
\hline $\mathrm{C}\left(4^{\prime}\right)-\mathrm{H}\left(4^{\prime}\right)$ & $1.07(4)$ \\
\hline$C\left(5^{\prime}\right)-C\left(6^{\prime} A\right)$ & $1.519(13)$ \\
\hline$C\left(5^{\prime}\right)-C\left(6^{\prime} B\right)$ & $1.530(12)$ \\
\hline $\mathrm{C}\left(5^{\prime}\right)-\mathrm{H}\left(5^{\prime}\right)$ & $1.06(4)$ \\
\hline$C\left(7^{\prime}\right)-C\left(8^{\prime}\right)$ & $1.482(5)$ \\
\hline$C(7 ")-C(8 ")$ & $1.482(5)$ \\
\hline$C\left(8^{\prime}\right)-C\left(9^{\prime}\right)$ & $1.369(5)$ \\
\hline$C\left(8^{\prime}\right)-C\left(8^{\prime \prime}\right)$ & $1.399(5)$ \\
\hline$C(8 ")-C(9 ")$ & $1.377(5)$ \\
\hline$C\left(9^{\prime}\right)-C\left(10^{\prime}\right)$ & $1.392(5)$ \\
\hline $\mathrm{C}\left(9^{\prime}\right)-\mathrm{H}\left(9^{\prime}\right)$ & 0.9500 \\
\hline$C(9 ")-C(10 ")$ & $1.396(5)$ \\
\hline $\mathrm{C}(9 ")-\mathrm{H}(9 ")$ & 0.9500 \\
\hline$C\left(10^{\prime}\right)-C\left(10^{\prime \prime}\right)$ & $1.388(5)$ \\
\hline $\mathrm{C}\left(10^{\prime}\right)-\mathrm{H}\left(10^{\prime}\right)$ & 0.9500 \\
\hline $\mathrm{C}(10 ")-\mathrm{H}(10 ")$ & 0.9500 \\
\hline$C\left(11^{\prime}\right)-C\left(12^{\prime}\right)$ & $1.494(5)$ \\
\hline $\mathrm{C}\left(12^{\prime}\right)-\mathrm{H}(12 \mathrm{~A})$ & 0.9800 \\
\hline $\mathrm{C}\left(12^{\prime}\right)-\mathrm{H}(12 \mathrm{~B})$ & 0.9800 \\
\hline $\mathrm{C}\left(12^{\prime}\right)-\mathrm{H}(12 \mathrm{C})$ & 0.9800 \\
\hline$C\left(13^{\prime}\right)-C\left(14^{\prime}\right)$ & $1.484(6)$ \\
\hline $\mathrm{C}\left(14^{\prime}\right)-\mathrm{H}(14 \mathrm{~A})$ & 0.9800 \\
\hline $\mathrm{C}\left(14^{\prime}\right)-\mathrm{H}(14 \mathrm{~B})$ & 0.9800 \\
\hline
\end{tabular}




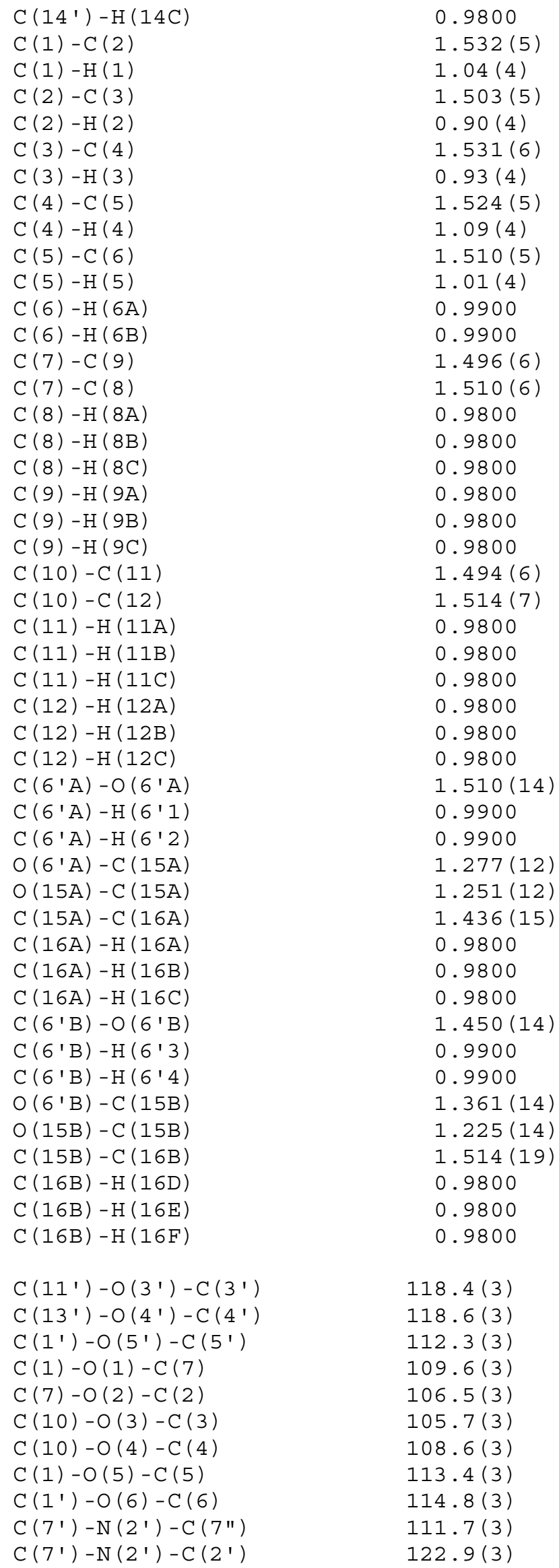




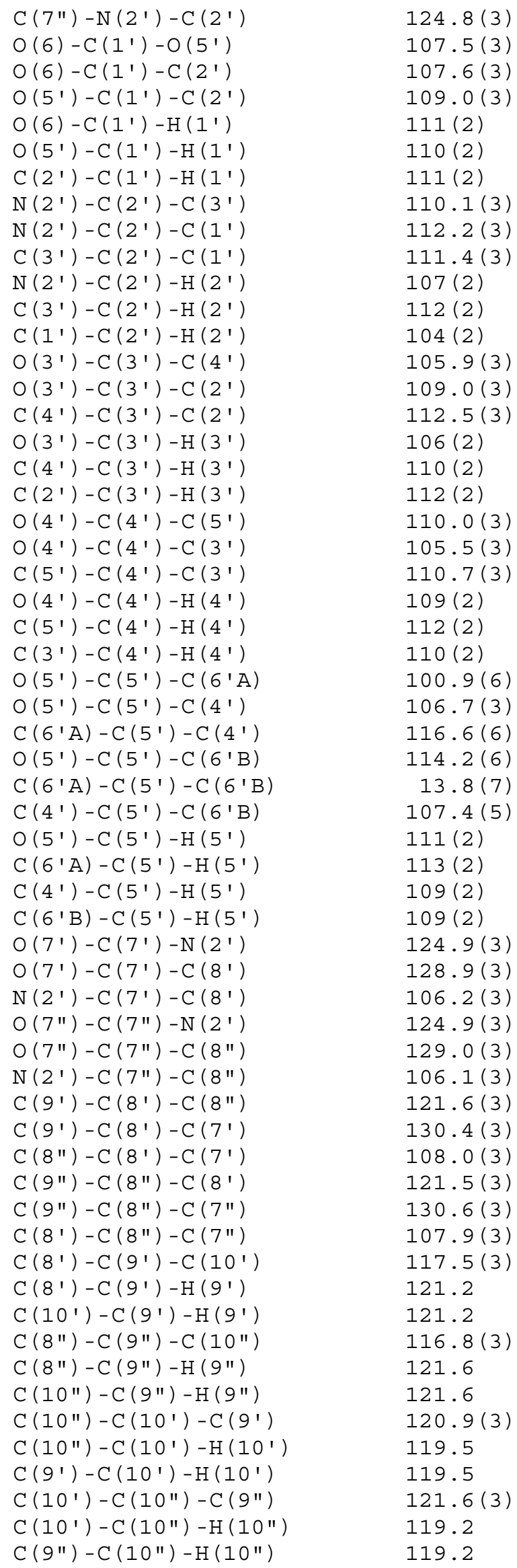




\begin{tabular}{|c|c|}
\hline $\mathrm{O}\left(11^{\prime}\right)-\mathrm{C}\left(11^{\prime}\right)-\mathrm{O}\left(3^{\prime}\right)$ & $123.2(3)$ \\
\hline $\mathrm{O}\left(11^{\prime}\right)-\mathrm{C}\left(11^{\prime}\right)-\mathrm{C}\left(12^{\prime}\right)$ & $126.1(4)$ \\
\hline $\mathrm{O}\left(3^{\prime}\right)-\mathrm{C}\left(11^{\prime}\right)-\mathrm{C}\left(12^{\prime}\right)$ & $110.7(3)$ \\
\hline $\mathrm{C}\left(11^{\prime}\right)-\mathrm{C}\left(12^{\prime}\right)-\mathrm{H}(12 \mathrm{~A})$ & 109.5 \\
\hline $\mathrm{C}\left(11^{\prime}\right)-\mathrm{C}\left(12^{\prime}\right)-\mathrm{H}(12 \mathrm{~B})$ & 109.5 \\
\hline $\mathrm{H}(12 \mathrm{~A})-\mathrm{C}\left(12^{\prime}\right)-\mathrm{H}(12 \mathrm{~B})$ & 109.5 \\
\hline $\mathrm{C}\left(11^{\prime}\right)-\mathrm{C}\left(12^{\prime}\right)-\mathrm{H}(12 \mathrm{C})$ & 109.5 \\
\hline $\mathrm{H}(12 \mathrm{~A})-\mathrm{C}\left(12^{\prime}\right)-\mathrm{H}(12 \mathrm{C})$ & 109.5 \\
\hline $\mathrm{H}(12 \mathrm{~B})-\mathrm{C}\left(12^{\prime}\right)-\mathrm{H}(12 \mathrm{C})$ & 109.5 \\
\hline $\mathrm{O}\left(13^{\prime}\right)-\mathrm{C}\left(13^{\prime}\right)-\mathrm{O}\left(4^{\prime}\right)$ & $123.1(4)$ \\
\hline $\mathrm{O}\left(13^{\prime}\right)-\mathrm{C}\left(13^{\prime}\right)-\mathrm{C}\left(14^{\prime}\right)$ & $126.5(4)$ \\
\hline $\mathrm{O}\left(4^{\prime}\right)-\mathrm{C}\left(13^{\prime}\right)-\mathrm{C}\left(14^{\prime}\right)$ & $110.5(3)$ \\
\hline $\mathrm{C}\left(13^{\prime}\right)-\mathrm{C}\left(14^{\prime}\right)-\mathrm{H}(14 \mathrm{~A})$ & 109.5 \\
\hline $\mathrm{C}\left(13^{\prime}\right)-\mathrm{C}\left(14^{\prime}\right)-\mathrm{H}(14 \mathrm{~B})$ & 109.5 \\
\hline $\mathrm{H}(14 \mathrm{~A})-\mathrm{C}\left(14^{\prime}\right)-\mathrm{H}(14 \mathrm{~B})$ & 109.5 \\
\hline $\mathrm{C}\left(13^{\prime}\right)-\mathrm{C}\left(14^{\prime}\right)-\mathrm{H}(14 \mathrm{C})$ & 109.5 \\
\hline $\mathrm{H}(14 \mathrm{~A})-\mathrm{C}\left(14^{\prime}\right)-\mathrm{H}(14 \mathrm{C})$ & 109.5 \\
\hline $\mathrm{H}(14 \mathrm{~B})-\mathrm{C}\left(14^{\prime}\right)-\mathrm{H}(14 \mathrm{C})$ & 109.5 \\
\hline $\mathrm{O}(5)-\mathrm{C}(1)-\mathrm{O}(1)$ & $109.2(3)$ \\
\hline $\mathrm{O}(5)-\mathrm{C}(1)-\mathrm{C}(2)$ & $114.2(3)$ \\
\hline $\mathrm{O}(1)-\mathrm{C}(1)-\mathrm{C}(2)$ & $104.5(3)$ \\
\hline $\mathrm{O}(5)-\mathrm{C}(1)-\mathrm{H}(1)$ & $99(2)$ \\
\hline $\mathrm{O}(1)-\mathrm{C}(1)-\mathrm{H}(1)$ & $110(2)$ \\
\hline $\mathrm{C}(2)-\mathrm{C}(1)-\mathrm{H}(1)$ & $120(2)$ \\
\hline $\mathrm{O}(2)-\mathrm{C}(2)-\mathrm{C}(3)$ & $108.2(3)$ \\
\hline $\mathrm{O}(2)-\mathrm{C}(2)-\mathrm{C}(1)$ & $103.3(3)$ \\
\hline$C(3)-C(2)-C(1)$ & $115.4(3)$ \\
\hline $\mathrm{O}(2)-\mathrm{C}(2)-\mathrm{H}(2)$ & $112(3)$ \\
\hline $\mathrm{C}(3)-\mathrm{C}(2)-\mathrm{H}(2)$ & $111(3)$ \\
\hline $\mathrm{C}(1)-\mathrm{C}(2)-\mathrm{H}(2)$ & $107(3)$ \\
\hline$O(3)-C(3)-C(2)$ & $107.0(3)$ \\
\hline$O(3)-C(3)-C(4)$ & $104.9(3)$ \\
\hline$C(2)-C(3)-C(4)$ & $115.4(3)$ \\
\hline $\mathrm{O}(3)-\mathrm{C}(3)-\mathrm{H}(3)$ & $108(2)$ \\
\hline$C(2)-C(3)-H(3)$ & $109(2)$ \\
\hline $\mathrm{C}(4)-\mathrm{C}(3)-\mathrm{H}(3)$ & $112(3)$ \\
\hline$O(4)-C(4)-C(5)$ & $109.9(3)$ \\
\hline$O(4)-C(4)-C(3)$ & $103.2(3)$ \\
\hline$C(5)-C(4)-C(3)$ & $111.9(3)$ \\
\hline $\mathrm{O}(4)-\mathrm{C}(4)-\mathrm{H}(4)$ & $113(2)$ \\
\hline $\mathrm{C}(5)-\mathrm{C}(4)-\mathrm{H}(4)$ & $107(2)$ \\
\hline $\mathrm{C}(3)-\mathrm{C}(4)-\mathrm{H}(4)$ & $112(2)$ \\
\hline$O(5)-C(5)-C(6)$ & $106.2(3)$ \\
\hline$O(5)-C(5)-C(4)$ & $110.2(3)$ \\
\hline$C(6)-C(5)-C(4)$ & $113.0(3)$ \\
\hline $\mathrm{O}(5)-\mathrm{C}(5)-\mathrm{H}(5)$ & $111(2)$ \\
\hline$C(6)-C(5)-H(5)$ & $109(2)$ \\
\hline$C(4)-C(5)-H(5)$ & $107(2)$ \\
\hline$O(6)-C(6)-C(5)$ & $109.6(3)$ \\
\hline $\mathrm{O}(6)-\mathrm{C}(6)-\mathrm{H}(6 \mathrm{~A})$ & 109.7 \\
\hline$C(5)-C(6)-H(6 A)$ & 109.7 \\
\hline $\mathrm{O}(6)-\mathrm{C}(6)-\mathrm{H}(6 \mathrm{~B})$ & 109.7 \\
\hline $\mathrm{C}(5)-\mathrm{C}(6)-\mathrm{H}(6 \mathrm{~B})$ & 109.7 \\
\hline $\mathrm{H}(6 \mathrm{~A})-\mathrm{C}(6)-\mathrm{H}(6 \mathrm{~B})$ & 108.2 \\
\hline $\mathrm{O}(2)-\mathrm{C}(7)-\mathrm{O}(1)$ & $104.4(3)$ \\
\hline $\mathrm{O}(2)-\mathrm{C}(7)-\mathrm{C}(9)$ & $109.0(3)$ \\
\hline $\mathrm{O}(1)-\mathrm{C}(7)-\mathrm{C}(9)$ & $109.6(3)$ \\
\hline $\mathrm{O}(2)-\mathrm{C}(7)-\mathrm{C}(8)$ & $111.1(3)$ \\
\hline$O(1)-C(7)-C(8)$ & $110.4(3)$ \\
\hline
\end{tabular}




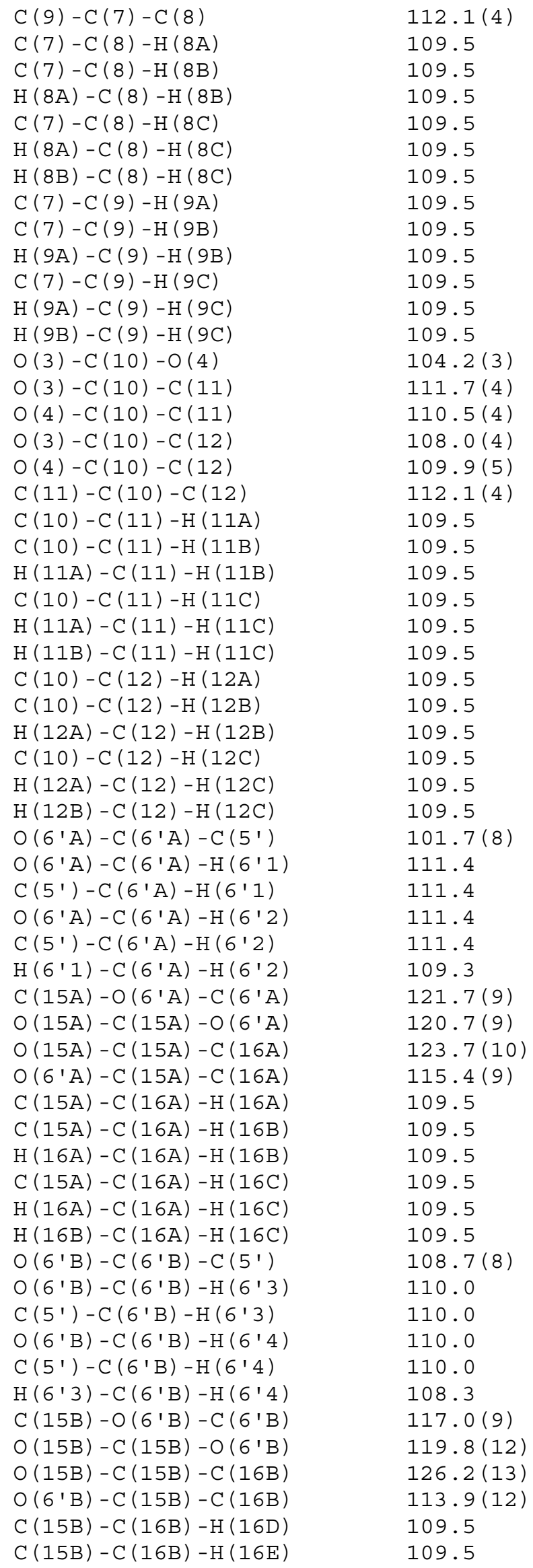


$\mathrm{H}(16 \mathrm{D})-\mathrm{C}(16 \mathrm{~B})-\mathrm{H}(16 \mathrm{E})$

109.5

$\mathrm{C}(15 \mathrm{~B})-\mathrm{C}(16 \mathrm{~B})-\mathrm{H}(16 \mathrm{~F})$

109.5

$\mathrm{H}(16 \mathrm{D})-\mathrm{C}(16 \mathrm{~B})-\mathrm{H}(16 \mathrm{~F})$

109.5

$\mathrm{H}(16 \mathrm{E})-\mathrm{C}(16 \mathrm{~B})-\mathrm{H}(16 \mathrm{~F})$

109.5

Symmetry transformations used to generate equivalent atoms: 
Table S4. Anisotropic displacement parameters $\left(A^{2} \times 10^{3}\right)$ for 3 . The anisotropic displacement factor exponent takes the form: $-2 \mathrm{pi}^{2}\left[\mathrm{~h}^{2} \mathrm{a} *^{2} \mathrm{U} 11+\ldots+2 \mathrm{hk} a * b * \mathrm{U} 12\right]$

\begin{tabular}{|c|c|c|c|c|c|c|}
\hline & $\mathrm{U} 11$ & $\mathrm{U} 22$ & U33 & $\mathrm{U} 23$ & U13 & $\mathrm{U} 12$ \\
\hline$O\left(3^{\prime}\right)$ & $32(1)$ & $37(1)$ & $24(1)$ & $3(1)$ & $0(1)$ & $21(1)$ \\
\hline$O\left(4{ }^{\prime}\right)$ & $31(2)$ & $46(2)$ & $23(1)$ & $0(1)$ & $-2(1)$ & $6(1)$ \\
\hline$O\left(5^{\prime}\right)$ & $57(2)$ & $51(2)$ & $28(1)$ & $-10(1)$ & $-3(1)$ & $38(2)$ \\
\hline$O\left(7^{\prime}\right)$ & $33(1)$ & $43(2)$ & $33(1)$ & 7 (1) & $9(1)$ & $18(1)$ \\
\hline O (7") & $27(1)$ & $28(1)$ & $25(1)$ & $0(1)$ & $2(1)$ & $10(1)$ \\
\hline$O\left(11^{\prime}\right)$ & $55(2)$ & $61(2)$ & $29(2)$ & $8(1)$ & $7(1)$ & $42(2)$ \\
\hline$O\left(13^{\prime}\right)$ & $43(2)$ & $80(2)$ & $24(1)$ & $4(1)$ & $2(1)$ & $28(2)$ \\
\hline$O(1)$ & $44(2)$ & $40(2)$ & $38(1)$ & $-10(1)$ & $-16(1)$ & $28(1)$ \\
\hline$O(2)$ & $31(1)$ & $40(2)$ & $32(1)$ & 2 (1) & $-7(1)$ & $18(1)$ \\
\hline$O(3)$ & $44(2)$ & $37(2)$ & $80(2)$ & $13(2)$ & $13(2)$ & $20(1)$ \\
\hline $\mathrm{O}(4)$ & $104(3)$ & $56(2)$ & $56(2)$ & $-20(2)$ & $-37(2)$ & $58(2)$ \\
\hline$O(5)$ & $26(1)$ & $40(2)$ & $30(1)$ & $-3(1)$ & $-3(1)$ & $18(1)$ \\
\hline$O(6)$ & $41(2)$ & $56(2)$ & $32(1)$ & $3(1)$ & $1(1)$ & $36(1)$ \\
\hline $\mathrm{N}\left(2^{\prime}\right)$ & $26(2)$ & $33(2)$ & $19(1)$ & $0(1)$ & $-1(1)$ & $16(1)$ \\
\hline$C\left(1^{\prime}\right)$ & $41(2)$ & $43(2)$ & $22(2)$ & $-1(2)$ & $0(2)$ & $27(2)$ \\
\hline$C\left(2^{\prime}\right)$ & $26(2)$ & $36(2)$ & $17(2)$ & $-2(1)$ & $3(1)$ & $16(2)$ \\
\hline$C\left(3^{\prime}\right)$ & $32(2)$ & $32(2)$ & $17(2)$ & 1 (1) & $0(1)$ & $18(2)$ \\
\hline$C\left(4^{\prime}\right)$ & $32(2)$ & $36(2)$ & $23(2)$ & $0(2)$ & $4(2)$ & $13(2)$ \\
\hline$C\left(5^{\prime}\right)$ & $50(3)$ & $34(2)$ & $33(2)$ & $-5(2)$ & $-2(2)$ & $21(2)$ \\
\hline$C\left(7^{\prime}\right)$ & $22(2)$ & $33(2)$ & $22(2)$ & $4(2)$ & $0(1)$ & $17(2)$ \\
\hline$C(7 ")$ & $23(2)$ & $32(2)$ & $19(2)$ & $0(1)$ & $-1(1)$ & $17(2)$ \\
\hline$C\left(8^{\prime}\right)$ & $26(2)$ & $28(2)$ & $23(2)$ & $1(1)$ & $-1(1)$ & $15(2)$ \\
\hline$C(8 ")$ & $24(2)$ & $30(2)$ & $25(2)$ & $2(1)$ & $-1(1)$ & $16(2)$ \\
\hline$C\left(9^{\prime}\right)$ & $31(2)$ & $33(2)$ & $29(2)$ & $3(2)$ & $-4(2)$ & $14(2)$ \\
\hline C (9") & $29(2)$ & $36(2)$ & $24(2)$ & $1(2)$ & $1(2)$ & $16(2)$ \\
\hline$C\left(10^{\prime}\right)$ & $42(2)$ & $30(2)$ & $36(2)$ & $-10(2)$ & $-3(2)$ & $14(2)$ \\
\hline C (10") & $45(2)$ & $37(2)$ & $25(2)$ & $-4(2)$ & $5(2)$ & $21(2)$ \\
\hline$C\left(11^{\prime}\right)$ & $28(2)$ & $38(2)$ & $32(2)$ & $-2(2)$ & $-6(2)$ & $16(2)$ \\
\hline$C\left(12^{\prime}\right)$ & $37(2)$ & $33(2)$ & $38(2)$ & $6(2)$ & $-3(2)$ & $16(2)$ \\
\hline$C\left(13^{\prime}\right)$ & $37(2)$ & $41(2)$ & $24(2)$ & $-7(2)$ & $-4(2)$ & $11(2)$ \\
\hline$C(14 ')$ & $39(2)$ & $84(4)$ & $30(2)$ & $-6(2)$ & $-5(2)$ & $19(2)$ \\
\hline$C(1)$ & $29(2)$ & $47(2)$ & $24(2)$ & $-1(2)$ & $0(2)$ & $22(2)$ \\
\hline$C(2)$ & $32(2)$ & $40(2)$ & $25(2)$ & $6(2)$ & $0(2)$ & $19(2)$ \\
\hline$C(3)$ & $28(2)$ & $36(2)$ & $38(2)$ & $7(2)$ & $-4(2)$ & $15(2)$ \\
\hline$C(4)$ & $45(2)$ & $50(3)$ & $37(2)$ & $0(2)$ & $-5(2)$ & $31(2)$ \\
\hline C (5) & $29(2)$ & $43(2)$ & $35(2)$ & $0(2)$ & $-1(2)$ & $25(2)$ \\
\hline$C(6)$ & $57(3)$ & $58(3)$ & $31(2)$ & $3(2)$ & $0(2)$ & $48(2)$ \\
\hline $\mathrm{C}(7)$ & $39(2)$ & $41(2)$ & $32(2)$ & $-7(2)$ & $-13(2)$ & $24(2)$ \\
\hline$C(8)$ & $65(3)$ & $62(3)$ & $41(2)$ & $-11(2)$ & $-4(2)$ & $39(3)$ \\
\hline$C(9)$ & $50(3)$ & $38(2)$ & $42(2)$ & $-1(2)$ & $-8(2)$ & $20(2)$ \\
\hline$C(10)$ & $38(2)$ & $37(2)$ & $82(3)$ & $-3(2)$ & $-9(2)$ & $23(2)$ \\
\hline$C(11)$ & $52(3)$ & $43(3)$ & $77(3)$ & $19(2)$ & $1(2)$ & $29(2)$ \\
\hline$C(12)$ & $42(3)$ & $54(3)$ & $257(10)$ & $-41(5)$ & $-36(5)$ & $25(3)$ \\
\hline
\end{tabular}


Table S5. Hydrogen coordinates $\left(\times 10^{4}\right)$ and isotropic displacement parameters $\left(A^{2} \times 10^{3}\right)$ for 3 .

\begin{tabular}{|c|c|c|c|c|}
\hline & $\mathrm{x}$ & $\mathrm{Y}$ & z & $\mathrm{U}(\mathrm{eq})$ \\
\hline $\mathrm{H}\left(\mathrm{I}^{\prime}\right)$ & $1210(19)$ & $3585(19)$ & $-1060(40)$ & 39 \\
\hline H (2' ) & $1809(18)$ & 4697 (17) & $-2760(40)$ & 31 \\
\hline H ( $3^{\prime}$ ) & $443(18)$ & $4072(17)$ & $-2200(40)$ & 31 \\
\hline $\mathrm{H}\left(4^{\prime}\right)$ & $909(18)$ & $3924(18)$ & $-4720(40)$ & 39 \\
\hline $\mathrm{H}\left(5^{\prime}\right)$ & $410(20)$ & $2940(20)$ & $-2540(40)$ & 47 \\
\hline $\mathrm{H}\left(9^{\prime}\right)$ & 2512 & 6695 & 386 & 39 \\
\hline H ( $9 "$ ) & 741 & 4944 & 2533 & 36 \\
\hline H (10') & 2123 & 6851 & 2397 & 45 \\
\hline H (10") & 1245 & 5996 & 3430 & 42 \\
\hline $\mathrm{H}(12 \mathrm{~A})$ & 341 & 5796 & -3340 & 55 \\
\hline $\mathrm{H}(12 \mathrm{~B})$ & 365 & 5406 & -4598 & 55 \\
\hline $\mathrm{H}(12 \mathrm{C})$ & 1023 & 5928 & -3902 & 55 \\
\hline $\mathrm{H}(14 \mathrm{~A})$ & -935 & 3740 & -5284 & 84 \\
\hline $\mathrm{H}(14 \mathrm{~B})$ & -1143 & 3018 & -4844 & 84 \\
\hline $\mathrm{H}(14 \mathrm{C})$ & -1060 & 3204 & -6369 & 84 \\
\hline $\mathrm{H}(1)$ & $1330(20)$ & $3790(18)$ & $2660(40)$ & 38 \\
\hline $\mathrm{H}(2)$ & 2111 (19) & 3886 (19) & $4170(40)$ & 39 \\
\hline $\mathrm{H}(3)$ & $2880(20)$ & 3604 (19) & $3480(40)$ & 42 \\
\hline $\mathrm{H}(4)$ & $3140(20)$ & 3676 (19) & $1290(40)$ & 49 \\
\hline H ( 5) & 2725 (19) & $4320(20)$ & $770(40)$ & 39 \\
\hline $\mathrm{H}(6 \mathrm{~A})$ & 2667 & 3648 & -1068 & 49 \\
\hline $\mathrm{H}(6 \mathrm{~B})$ & 1906 & 3202 & -793 & 49 \\
\hline $\mathrm{H}(8 \mathrm{~A})$ & 2257 & 5545 & 4173 & 80 \\
\hline $\mathrm{H}(8 \mathrm{~B})$ & 2759 & 5416 & 4974 & 80 \\
\hline $\mathrm{H}(8 \mathrm{C})$ & 2035 & 4838 & 4740 & 80 \\
\hline $\mathrm{H}(9 \mathrm{~A})$ & 3238 & 5509 & 1583 & 67 \\
\hline $\mathrm{H}(9 \mathrm{~B})$ & 3488 & 5880 & 2954 & 67 \\
\hline $\mathrm{H}(9 \mathrm{C})$ & 2933 & 5923 & 2157 & 67 \\
\hline $\mathrm{H}(11 \mathrm{~A})$ & 2995 & 2694 & 2882 & 82 \\
\hline $\mathrm{H}(11 \mathrm{~B})$ & 2573 & 1980 & 2293 & 82 \\
\hline $\mathrm{H}(11 \mathrm{C})$ & 2470 & 2123 & 3783 & 82 \\
\hline $\mathrm{H}(12 \mathrm{~A})$ & 1115 & 1864 & 2996 & 176 \\
\hline $\mathrm{H}(12 \mathrm{~B})$ & 1445 & 1472 & 2429 & 176 \\
\hline $\mathrm{H}(12 \mathrm{C})$ & 1228 & 1854 & 1449 & 176 \\
\hline $\mathrm{H}\left(\sigma^{\prime} 1\right)$ & 667 & 2808 & -5250 & 50 \\
\hline $\mathrm{H}\left(6^{\prime} 2\right)$ & 1074 & 2613 & -4270 & 50 \\
\hline $\mathrm{H}(16 \mathrm{~A})$ & -491 & 794 & -2486 & 113 \\
\hline $\mathrm{H}(16 \mathrm{~B})$ & -819 & 1038 & -3541 & 113 \\
\hline $\mathrm{H}(16 \mathrm{C})$ & -778 & 1244 & -2032 & 113 \\
\hline $\mathrm{H}\left(6{ }^{\prime} 3\right)$ & 105 & 2536 & -4780 & 61 \\
\hline $\mathrm{H}\left(6^{\prime} 4^{\prime}\right)$ & 854 & 2854 & -5179 & 61 \\
\hline $\mathrm{H}(16 \mathrm{D})$ & -31 & 588 & -4535 & 214 \\
\hline $\mathrm{H}(16 \mathrm{E})$ & 81 & 936 & -3139 & 214 \\
\hline $\mathrm{H}(16 \mathrm{~F})$ & 688 & 1030 & -3982 & 214 \\
\hline
\end{tabular}


Table S6. Torsion angles [deg] for 3.

\begin{tabular}{|c|c|}
\hline$C(6)-O(6)-C\left(1^{\prime}\right)-O\left(5^{\prime}\right)$ & $-81.5(3)$ \\
\hline$C(6)-O(6)-C\left(1^{\prime}\right)-C\left(2^{\prime}\right)$ & $161.2(3)$ \\
\hline$C\left(5^{\prime}\right)-O\left(5^{\prime}\right)-C\left(1^{\prime}\right)-O(6)$ & $177.5(3)$ \\
\hline$C\left(5^{\prime}\right)-O\left(5^{\prime}\right)-C\left(1^{\prime}\right)-C\left(2^{\prime}\right)$ & $-66.1(4)$ \\
\hline $\mathrm{C}\left(7^{\prime}\right)-\mathrm{N}\left(2^{\prime}\right)-\mathrm{C}\left(2^{\prime}\right)-\mathrm{C}\left(3^{\prime}\right)$ & $-110.8(3)$ \\
\hline $\mathrm{C}\left(7^{\prime \prime}\right)-\mathrm{N}\left(2^{\prime}\right)-\mathrm{C}\left(2^{\prime}\right)-\mathrm{C}\left(3^{\prime}\right)$ & $59.5(4)$ \\
\hline $\mathrm{C}\left(7^{\prime}\right)-\mathrm{N}\left(2^{\prime}\right)-\mathrm{C}\left(2^{\prime}\right)-\mathrm{C}\left(1^{\prime}\right)$ & $124.5(3)$ \\
\hline $\mathrm{C}\left(7^{\prime \prime}\right)-\mathrm{N}\left(2^{\prime}\right)-\mathrm{C}\left(2^{\prime}\right)-\mathrm{C}\left(1^{\prime}\right)$ & $-65.2(4)$ \\
\hline $\mathrm{O}(6)-\mathrm{C}\left(1^{\prime}\right)-\mathrm{C}\left(2^{\prime}\right)-\mathrm{N}\left(2^{\prime}\right)$ & $-67.9(4)$ \\
\hline $\mathrm{O}\left(5^{\prime}\right)-\mathrm{C}\left(1^{\prime}\right)-\mathrm{C}\left(2^{\prime}\right)-\mathrm{N}\left(2^{\prime}\right)$ & $175.8(3)$ \\
\hline$O(6)-C\left(1^{\prime}\right)-C\left(2^{\prime}\right)-C\left(3^{\prime}\right)$ & $168.1(3)$ \\
\hline $\mathrm{O}\left(5^{\prime}\right)-\mathrm{C}\left(\mathrm{I}^{\prime}\right)-\mathrm{C}\left(2^{\prime}\right)-\mathrm{C}\left(3^{\prime}\right)$ & $51.8(4)$ \\
\hline$C\left(11^{\prime}\right)-O\left(3^{\prime}\right)-C\left(3^{\prime}\right)-C\left(4^{\prime}\right)$ & $138.8(3)$ \\
\hline$C\left(11^{\prime}\right)-O\left(3^{\prime}\right)-C\left(3^{\prime}\right)-C\left(2^{\prime}\right)$ & $-99.9(3)$ \\
\hline $\mathrm{N}\left(2^{\prime}\right)-\mathrm{C}\left(2^{\prime}\right)-\mathrm{C}\left(3^{\prime}\right)-\mathrm{O}\left(3^{\prime}\right)$ & $72.4(3)$ \\
\hline$C\left(1^{\prime}\right)-C\left(2^{\prime}\right)-C\left(3^{\prime}\right)-O\left(3^{\prime}\right)$ & $-162.4(3)$ \\
\hline$N\left(2^{\prime}\right)-C\left(2^{\prime}\right)-C\left(3^{\prime}\right)-C\left(4^{\prime}\right)$ & $-170.5(3)$ \\
\hline$C\left(1^{\prime}\right)-C\left(2^{\prime}\right)-C\left(3^{\prime}\right)-C\left(4^{\prime}\right)$ & $-45.3(4)$ \\
\hline$C\left(13^{\prime}\right)-O\left(4^{\prime}\right)-C\left(4^{\prime}\right)-C\left(5^{\prime}\right)$ & $-123.8(4)$ \\
\hline$C\left(13^{\prime}\right)-O\left(4^{\prime}\right)-C\left(4^{\prime}\right)-C\left(3^{\prime}\right)$ & $116.7(3)$ \\
\hline $\mathrm{O}\left(3^{\prime}\right)-\mathrm{C}\left(3^{\prime}\right)-\mathrm{C}\left(4^{\prime}\right)-\mathrm{O}\left(4^{\prime}\right)$ & $-73.0(3)$ \\
\hline$C\left(2^{\prime}\right)-C\left(3^{\prime}\right)-C\left(4^{\prime}\right)-O\left(4^{\prime}\right)$ & $168.0(3)$ \\
\hline$O\left(3^{\prime}\right)-C\left(3^{\prime}\right)-C\left(4^{\prime}\right)-C\left(5^{\prime}\right)$ & $168.0(3)$ \\
\hline$C\left(2^{\prime}\right)-C\left(3^{\prime}\right)-C\left(4^{\prime}\right)-C\left(5^{\prime}\right)$ & $48.9(4)$ \\
\hline$C\left(1^{\prime}\right)-O\left(5^{\prime}\right)-C\left(5^{\prime}\right)-C\left(6^{\prime} A\right)$ & $-168.6(5)$ \\
\hline$C\left(1^{\prime}\right)-O\left(5^{\prime}\right)-C\left(5^{\prime}\right)-C\left(4^{\prime}\right)$ & $69.1(4)$ \\
\hline$C\left(1^{\prime}\right)-O\left(5^{\prime}\right)-C\left(5^{\prime}\right)-C\left(6^{\prime} B\right)$ & $-172.4(5)$ \\
\hline$O\left(4^{\prime}\right)-C\left(4^{\prime}\right)-C\left(5^{\prime}\right)-O\left(5^{\prime}\right)$ & $-174.7(3)$ \\
\hline$C\left(3^{\prime}\right)-C\left(4^{\prime}\right)-C\left(5^{\prime}\right)-O\left(5^{\prime}\right)$ & $-58.4(4)$ \\
\hline$O\left(4^{\prime}\right)-C\left(4^{\prime}\right)-C\left(5^{\prime}\right)-C\left(6^{\prime} A\right)$ & $73.6(7)$ \\
\hline$C\left(3^{\prime}\right)-C\left(4^{\prime}\right)-C\left(5^{\prime}\right)-C\left(6^{\prime} A\right)$ & $-170.2(6)$ \\
\hline$O\left(4^{\prime}\right)-C\left(4^{\prime}\right)-C\left(5^{\prime}\right)-C\left(6^{\prime} B\right)$ & $62.5(6)$ \\
\hline$C\left(3^{\prime}\right)-C\left(4^{\prime}\right)-C\left(5^{\prime}\right)-C\left(6^{\prime} B\right)$ & $178.8(6)$ \\
\hline $\mathrm{C}\left(7^{\prime \prime}\right)-\mathrm{N}\left(2^{\prime}\right)-\mathrm{C}\left(7^{\prime}\right)-\mathrm{O}\left(7^{\prime}\right)$ & $-176.0(3)$ \\
\hline $\mathrm{C}\left(2^{\prime}\right)-\mathrm{N}\left(2^{\prime}\right)-\mathrm{C}\left(7^{\prime}\right)-\mathrm{O}\left(7^{\prime}\right)$ & $-4.6(5)$ \\
\hline$C\left(7^{\prime \prime}\right)-N\left(2^{\prime}\right)-C\left(7^{\prime}\right)-C\left(8^{\prime}\right)$ & $2.3(3)$ \\
\hline $\mathrm{C}\left(2^{\prime}\right)-\mathrm{N}\left(2^{\prime}\right)-\mathrm{C}\left(7^{\prime}\right)-\mathrm{C}\left(8^{\prime}\right)$ & $173.7(3)$ \\
\hline $\mathrm{C}\left(7^{\prime}\right)-\mathrm{N}\left(2^{\prime}\right)-\mathrm{C}\left(7^{\prime \prime}\right)-\mathrm{O}\left(7^{\prime \prime}\right)$ & $178.2(3)$ \\
\hline $\mathrm{C}\left(2^{\prime}\right)-\mathrm{N}\left(2^{\prime}\right)-\mathrm{C}\left(7^{\prime \prime}\right)-\mathrm{O}\left(7^{\prime \prime}\right)$ & $6.9(5)$ \\
\hline $\mathrm{C}\left(7^{\prime}\right)-\mathrm{N}\left(2^{\prime}\right)-\mathrm{C}\left(7^{\prime \prime}\right)-\mathrm{C}\left(8^{\prime \prime}\right)$ & $-1.7(3)$ \\
\hline $\mathrm{C}\left(2^{\prime}\right)-\mathrm{N}\left(2^{\prime}\right)-\mathrm{C}\left(7^{\prime \prime}\right)-\mathrm{C}\left(8^{\prime \prime}\right)$ & $-173.0(3)$ \\
\hline$O\left(7^{\prime}\right)-C\left(7^{\prime}\right)-C\left(8^{\prime}\right)-C\left(9^{\prime}\right)$ & $-4.3(6)$ \\
\hline$N\left(2^{\prime}\right)-C\left(7^{\prime}\right)-C\left(8^{\prime}\right)-C\left(9^{\prime}\right)$ & $177.6(3)$ \\
\hline$O\left(7^{\prime}\right)-C\left(7^{\prime}\right)-C\left(8^{\prime}\right)-C\left(8^{\prime \prime}\right)$ & $176.2(3)$ \\
\hline$N\left(2^{\prime}\right)-C\left(7^{\prime}\right)-C\left(8^{\prime}\right)-C\left(8^{\prime \prime}\right)$ & $-2.0(3)$ \\
\hline$C\left(9^{\prime}\right)-C\left(8^{\prime}\right)-C\left(8^{\prime \prime}\right)-C\left(9^{\prime \prime}\right)$ & $-0.5(5)$ \\
\hline$C\left(7^{\prime}\right)-C\left(8^{\prime}\right)-C\left(8^{\prime \prime}\right)-C\left(9^{\prime \prime}\right)$ & $179.1(3)$ \\
\hline$C\left(9^{\prime}\right)-C\left(8^{\prime}\right)-C\left(8^{\prime \prime}\right)-C\left(7^{\prime \prime}\right)$ & $-178.6(3)$ \\
\hline$C\left(7^{\prime}\right)-C\left(8^{\prime}\right)-C\left(8^{\prime \prime}\right)-C\left(7^{\prime \prime}\right)$ & $0.9(4)$ \\
\hline$O(7 ")-C(7 ")-C(8 ")-C(9 ")$ & $2.6(6)$ \\
\hline$N\left(2^{\prime}\right)-C(7 ")-C(8 ")-C\left(9^{\prime \prime}\right)$ & $-177.5(3)$ \\
\hline$O(7 ")-C(7 ")-C(8 ")-C(8 ')$ & $-179.5(3)$ \\
\hline $\mathrm{N}\left(2^{\prime}\right)-\mathrm{C}\left(7^{\prime \prime}\right)-\mathrm{C}\left(8^{\prime \prime}\right)-\mathrm{C}\left(8^{\prime}\right)$ & $0.4(3)$ \\
\hline$C\left(8^{\prime \prime}\right)-C\left(8^{\prime}\right)-C\left(9^{\prime}\right)-C\left(10^{\prime}\right)$ & $-1.1(5)$ \\
\hline$C\left(7^{\prime}\right)-C\left(8^{\prime}\right)-C\left(9^{\prime}\right)-C\left(10^{\prime}\right)$ & $179.5(3)$ \\
\hline$C\left(8^{\prime}\right)-C\left(8^{\prime \prime}\right)-C\left(9^{\prime \prime}\right)-C\left(10^{\prime \prime}\right)$ & $2.3(5)$ \\
\hline
\end{tabular}




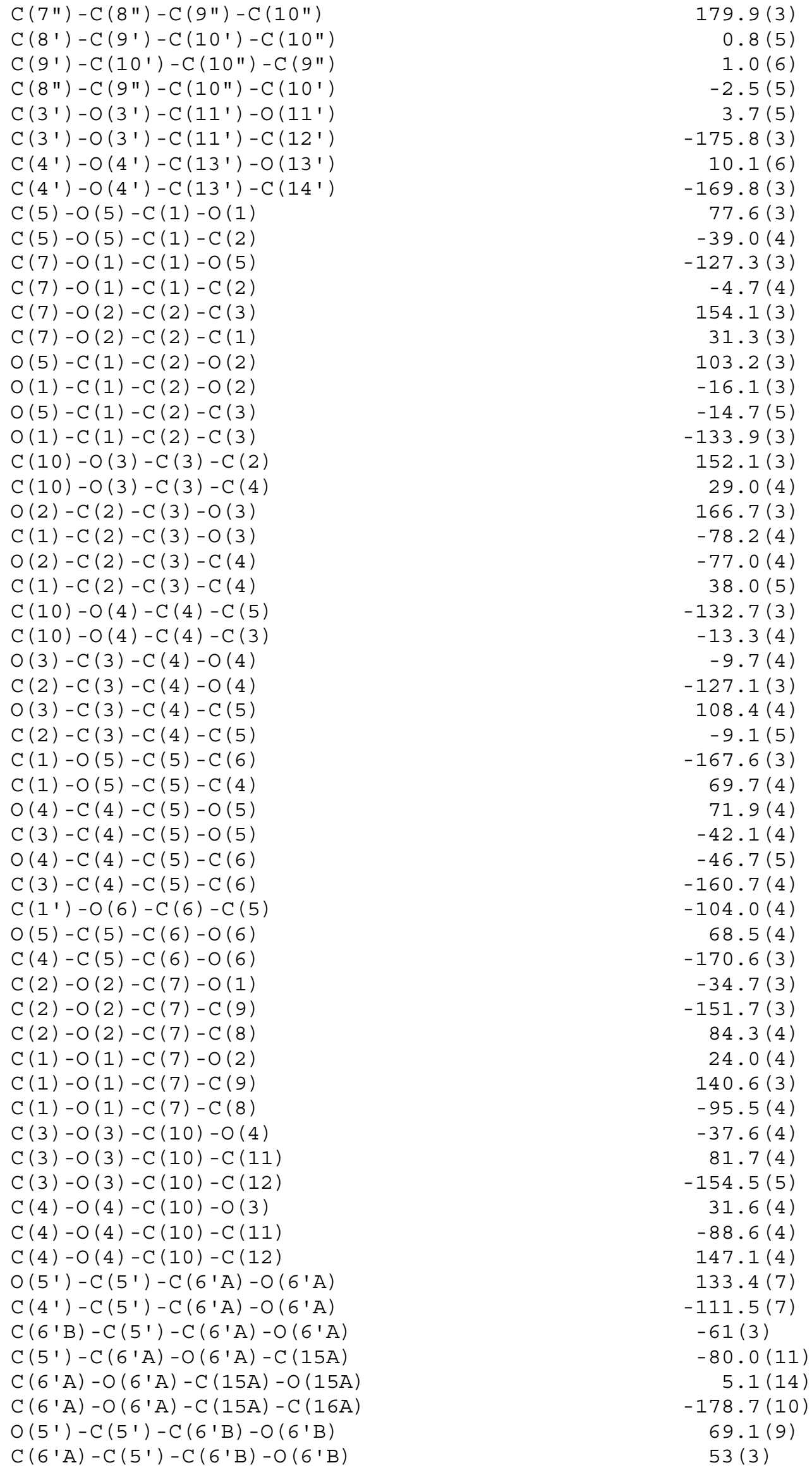


$C\left(4^{\prime}\right)-C\left(5^{\prime}\right)-C\left(6^{\prime} B\right)-O\left(6^{\prime} B\right)$

$-172.7(7)$

$C\left(5^{\prime}\right)-C\left(6^{\prime} B\right)-O\left(6^{\prime} B\right)-C(15 B)$

$168.6(8)$

$C\left(6^{\prime} B\right)-O\left(6^{\prime} B\right)-C(15 B)-O(15 B)$

$8.8(17)$

$C\left(6^{\prime} B\right)-O\left(6^{\prime} B\right)-C(15 B)-C(16 B)$

$-173.8(11)$

Symmetry transformations used to generate equivalent atoms: 
Table S7. XYZ file for 3 (orientation A).

\begin{tabular}{|c|c|c|c|}
\hline \multicolumn{4}{|l|}{87} \\
\hline \multicolumn{4}{|c|}{ XYZ file for : $3 \mathrm{~A}$} \\
\hline 0 & -0.08838 & 2.97704 & -2.41029 \\
\hline $\mathrm{H}$ & 0.88522 & -2.52931 & -2.36198 \\
\hline $\mathrm{H}$ & 3.83118 & 1.22487 & -2.19533 \\
\hline $\mathrm{H}$ & 5.30308 & 1.81309 & -1.97452 \\
\hline $\mathrm{H}$ & 3.44876 & -3.01973 & -1.99020 \\
\hline $\mathrm{H}$ & 2.04638 & 4.95224 & -1.65280 \\
\hline $\mathrm{H}$ & -1.47402 & 1.03212 & -1.82570 \\
\hline C & 4.41138 & 1.71874 & -1.57907 \\
\hline 0 & 0.02098 & -0.83710 & -1.71711 \\
\hline $\mathrm{H}$ & 2.57435 & -0.96040 & -1.59855 \\
\hline $\mathrm{H}$ & 4.03154 & 2.60706 & -1.41408 \\
\hline $\mathrm{C}$ & 0.80068 & -2.03302 & -1.50952 \\
\hline $\mathrm{H}$ & -3.27140 & 4.94583 & -1.15489 \\
\hline $\mathrm{H}$ & -4.60778 & -2.18492 & -1.20684 \\
\hline $\mathrm{C}$ & 0.26567 & 2.77513 & -1.26339 \\
\hline $\mathrm{H}$ & -4.13203 & 0.30226 & -1.17412 \\
\hline $\mathrm{H}$ & 4.69929 & -5.15244 & -0.84129 \\
\hline $\mathrm{C}$ & 3.13408 & -2.84275 & -0.96653 \\
\hline 0 & -2.07605 & -1.38936 & -1.05870 \\
\hline $\mathrm{C}$ & 2.17152 & -1.66243 & -0.99695 \\
\hline 0 & -6.27725 & 0.75492 & -0.68689 \\
\hline $\mathrm{C}$ & 2.04713 & 4.58816 & -0.77549 \\
\hline $\mathrm{H}$ & 0.34580 & -2.61910 & -0.85398 \\
\hline $\mathrm{C}$ & -1.32386 & 0.88296 & -0.84281 \\
\hline $\mathrm{H}$ & 3.73916 & -6.32816 & -0.33414 \\
\hline 0 & 4.96984 & -0.35179 & -0.55053 \\
\hline $\mathrm{C}$ & -0.93791 & -0.59140 & -0.74758 \\
\hline $\mathrm{H}$ & 5.12207 & -2.82916 & -0.42870 \\
\hline $\mathrm{H}$ & -4.56382 & 4.52615 & -0.30962 \\
\hline 0 & -5.92901 & -4.29243 & -0.15821 \\
\hline $\mathrm{H}$ & -3.74063 & -6.30173 & -0.13521 \\
\hline 0 & -3.12871 & 2.44096 & -0.45533 \\
\hline $\mathrm{C}$ & -3.60769 & 4.73476 & -0.25884 \\
\hline 0 & 2.49386 & -3.97656 & -0.37687 \\
\hline C & 1.24167 & 3.53178 & -0.44477 \\
\hline C & 4.26713 & -5.55056 & -0.05707 \\
\hline C & -4.27908 & -2.22878 & -0.27413 \\
\hline C & 4.51546 & 0.97284 & -0.28609 \\
\hline $\mathrm{N}$ & -0.23259 & 1.76707 & -0.44156 \\
\hline $\mathrm{H}$ & 3.42885 & 5.85001 & 0.02640 \\
\hline C & -3.66894 & 0.15153 & -0.22709 \\
\hline C & 4.32133 & -2.58758 & -0.03366 \\
\hline $\mathrm{C}$ & -4.76736 & -4.53823 & 0.14464 \\
\hline 0 & -3.81781 & -3.57068 & 0.02551 \\
\hline $\mathrm{C}$ & -3.11353 & -1.25623 & -0.08500 \\
\hline C & 2.86722 & 5.10921 & 0.22179 \\
\hline C & -2.57311 & 1.18489 & -0.02127 \\
\hline $\mathrm{H}$ & -3.47214 & 5.50538 & 0.33094 \\
\hline $\mathrm{H}$ & 6.33873 & 1.66646 & 0.36243 \\
\hline $\mathrm{H}$ & 4.95152 & -5.83227 & 0.58520 \\
\hline $\mathrm{H}$ & -5.02415 & -1.98486 & 0.33044 \\
\hline C & -2.86876 & 3.55519 & 0.28486 \\
\hline C & -4.22561 & -5.87584 & 0.60244 \\
\hline C & -5.88546 & 0.78710 & 0.44615 \\
\hline 0 & 3.21251 & 0.79652 & 0.27044 \\
\hline
\end{tabular}




$\begin{array}{rrrr}\mathrm{H} & -0.59304 & -0.82893 & 0.18692 \\ \mathrm{H} & -4.96843 & -6.45152 & 0.88015 \\ \mathrm{O} & 1.97688 & -1.14871 & 0.32620 \\ \mathrm{C} & 3.36940 & -4.54133 & 0.58251 \\ \mathrm{C} & 4.43593 & -1.18544 & 0.49600 \\ \mathrm{C} & 5.41914 & 1.67171 & 0.70108 \\ \mathrm{H} & 5.11991 & 2.59747 & 0.81941 \\ \mathrm{O} & -4.63824 & 0.40577 & 0.80686 \\ \mathrm{C} & 1.25345 & 2.97604 & 0.83865 \\ \mathrm{C} & 3.10926 & -0.50364 & 0.84582 \\ \mathrm{H} & -2.73206 & -1.36957 & 0.89389 \\ \mathrm{O} & 4.09648 & -3.43541 & 1.08255 \\ \mathrm{C} & 0.30116 & 1.84125 & 0.85133 \\ \mathrm{H} & -2.38481 & 1.28658 & 0.89365 \\ \mathrm{H} & -3.61688 & -5.74059 & 1.35842 \\ \mathrm{H} & -7.60846 & 1.05986 & 1.48949 \\ \mathrm{H} & 4.94817 & -1.16838 & 1.23327 \\ \mathrm{O} & -2.14401 & 3.55269 & 1.24894 \\ \mathrm{C} & -6.65508 & 1.23528 & 1.63304 \\ \mathrm{C} & 2.87606 & 4.56161 & 1.49675 \\ \mathrm{H} & 5.38534 & 1.20498 & 1.56212 \\ \mathrm{H} & -6.51641 & 2.19576 & 1.76869 \\ \mathrm{H} & 2.70181 & -6.10263 & 1.74811 \\ \mathrm{H} & 1.61891 & -4.93466 & 1.59396 \\ \mathrm{C} & 2.56993 & -5.13194 & 1.72460 \\ \mathrm{C} & 2.08371 & 3.45998 & 1.82552 \\ \mathrm{O} & 0.00657 & 1.09805 & 1.76907 \\ \mathrm{H} & 2.81290 & -0.42656 & 1.84482 \\ \mathrm{H} & 3.43483 & 4.94590 & 2.16221 \\ \mathrm{H} & -6.34868 & 0.74604 & 2.42500 \\ \mathrm{H} & 2.87144 & -4.74065 & 2.57099 \\ \mathrm{H} & 2.11568 & 3.06328 & 2.68810\end{array}$


Table S8. XYZ file for 3 (orientation B).

\begin{tabular}{|c|c|c|c|}
\hline \multicolumn{4}{|l|}{87} \\
\hline \multicolumn{4}{|c|}{ YZ file for : $3 B$} \\
\hline $\mathrm{H}$ & 0.96533 & 3.89530 & -3.83592 \\
\hline 0 & -0.70430 & 1.41673 & -3.51738 \\
\hline $\mathrm{H}$ & -4.25363 & 3.07931 & -3.04634 \\
\hline $\mathrm{H}$ & 4.79652 & 1.63171 & -2.96299 \\
\hline $\mathrm{H}$ & 2.08977 & 5.68916 & -2.80897 \\
\hline $\mathrm{C}$ & 1.01462 & 3.96232 & -2.88971 \\
\hline $\mathrm{H}$ & 3.48056 & 0.73380 & -2.81040 \\
\hline $\mathrm{H}$ & 3.37546 & 2.32837 & -2.72519 \\
\hline C & 3.93135 & 1.55039 & -2.50990 \\
\hline C & 1.68393 & 5.01688 & -2.27445 \\
\hline $\mathrm{C}$ & -0.34953 & 1.81299 & -2.42257 \\
\hline C & -4.56572 & 3.22349 & -2.12855 \\
\hline $\mathrm{H}$ & -5.45821 & 2.83313 & -2.02197 \\
\hline $\mathrm{H}$ & -4.60536 & 4.18500 & -1.94416 \\
\hline C & 0.42994 & 3.02568 & -2.08044 \\
\hline $\mathrm{H}$ & -1.68481 & -0.29959 & -2.05544 \\
\hline 0 & -3.62813 & 1.21849 & -1.33456 \\
\hline C & -3.61963 & 2.57156 & -1.17308 \\
\hline $\mathrm{H}$ & 2.67433 & -1.16296 & -1.24086 \\
\hline $\mathrm{H}$ & 5.77357 & 2.73216 & -0.86780 \\
\hline 0 & 0.15306 & -1.60136 & -1.23786 \\
\hline $\mathrm{H}$ & 1.35912 & -3.20163 & -1.13344 \\
\hline $\mathrm{N}$ & -0.65719 & 1.19434 & -1.21324 \\
\hline $\mathrm{C}$ & 4.14706 & 1.48557 & -1.03062 \\
\hline $\mathrm{C}$ & 1.76689 & 5.09956 & -0.89179 \\
\hline $\mathrm{H}$ & -4.15817 & -1.16737 & -0.98226 \\
\hline $\mathrm{C}$ & -1.53564 & 0.03127 & -1.11782 \\
\hline $\mathrm{H}$ & 4.37939 & 3.51365 & -0.78603 \\
\hline 0 & -6.36357 & -0.97134 & -0.60538 \\
\hline $\mathrm{H}$ & 3.95725 & -2.97173 & -0.74962 \\
\hline 0 & 4.86684 & 0.29569 & -0.71897 \\
\hline $\mathrm{H}$ & 2.21741 & 5.83756 & -0.49796 \\
\hline C & 4.86261 & 2.70777 & -0.50738 \\
\hline C & 0.51717 & 3.10552 & -0.68681 \\
\hline C & 1.15192 & -2.40766 & -0.57952 \\
\hline 0 & -2.93702 & 3.13398 & -0.35305 \\
\hline C & -2.84296 & 0.41573 & -0.43239 \\
\hline 0 & 2.89125 & 1.32366 & -0.37092 \\
\hline C & 2.40459 & -1.59173 & -0.36883 \\
\hline C & -0.86286 & -1.14485 & -0.41364 \\
\hline 0 & -1.80715 & -2.20180 & -0.27191 \\
\hline C & 1.20423 & 4.12493 & -0.06535 \\
\hline C & -3.70131 & -0.79308 & -0.09613 \\
\hline C & -0.18638 & 1.93174 & -0.11937 \\
\hline C & 3.58434 & -2.42693 & 0.11200 \\
\hline C & -6.01872 & -0.36861 & 0.37243 \\
\hline $\mathrm{H}$ & -4.40396 & -3.26353 & 0.32873 \\
\hline $\mathrm{H}$ & 5.51254 & -1.79344 & 0.46170 \\
\hline $\mathrm{H}$ & 4.89925 & 2.67094 & 0.47123 \\
\hline $\mathrm{H}$ & 0.80628 & -2.72110 & 0.29364 \\
\hline $\mathrm{H}$ & -2.95042 & -3.94741 & 0.49366 \\
\hline $\mathrm{H}$ & -2.70517 & 0.94402 & 0.33233 \\
\hline C & 4.48268 & -0.07805 & 0.61819 \\
\hline $\mathrm{H}$ & -6.95841 & 1.32646 & 0.98502 \\
\hline C & -2.87740 & -1.85738 & 0.61020 \\
\hline
\end{tabular}




$\begin{array}{lrrl}\mathrm{H} & -0.50385 & -0.87508 & 0.50668 \\ \mathrm{C} & 4.66862 & -1.56278 & 0.76201 \\ \mathrm{O} & -4.73097 & -0.30275 & 0.78284 \\ \mathrm{O} & 2.07270 & -0.59665 & 0.60695 \\ \mathrm{C} & -3.53671 & -3.21218 & 0.80337 \\ \mathrm{H} & 5.57941 & -4.09399 & 1.13224 \\ \mathrm{C} & 3.03629 & 0.41689 & 0.71943 \\ \mathrm{H} & -7.79027 & -0.00383 & 1.29992 \\ \mathrm{H} & 1.29109 & 4.16291 & 0.87987 \\ \mathrm{C} & -6.89641 & 0.39774 & 1.29127 \\ \mathrm{O} & 3.16971 & -3.28551 & 1.17691 \\ \mathrm{H} & 4.95976 & 0.36055 & 1.23970 \\ \mathrm{O} & -0.35084 & 1.62696 & 1.04744 \\ \mathrm{H} & -2.50889 & -1.45209 & 1.51382 \\ \mathrm{H} & 2.70219 & 0.86677 & 1.60134 \\ \mathrm{C} & 5.21440 & -4.18159 & 2.03742 \\ \mathrm{H} & 4.86238 & -5.08757 & 2.16183 \\ \mathrm{H} & -6.52019 & 0.37663 & 2.19595 \\ \mathrm{O} & 4.58792 & -1.85845 & 2.14814 \\ \mathrm{C} & 4.11360 & -3.18899 & 2.22843 \\ \mathrm{O} & -3.72428 & -3.24946 & 2.30168 \\ \mathrm{H} & 5.92302 & -4.01343 & 2.69305 \\ \mathrm{O} & -1.60871 & -3.73009 & 2.65200 \\ \mathrm{C} & -2.76616 & -3.54871 & 3.09111 \\ \mathrm{H} & 2.45052 & -3.42760 & 3.41972 \\ \mathrm{C} & 3.41768 & -3.35898 & 3.56227 \\ \mathrm{H} & 3.74208 & -4.17421 & 3.99853 \\ \mathrm{H} & 3.60979 & -2.58501 & 4.13188 \\ \mathrm{C} & -3.10179 & -3.57091 & 4.48753 \\ \mathrm{H} & -4.06909 & -3.69095 & 4.58918 \\ \mathrm{H} & -2.63298 & -4.31206 & 4.92447 \\ \mathrm{H} & -2.83250 & -2.72434 & 4.90171 \\ & & & \end{array}$




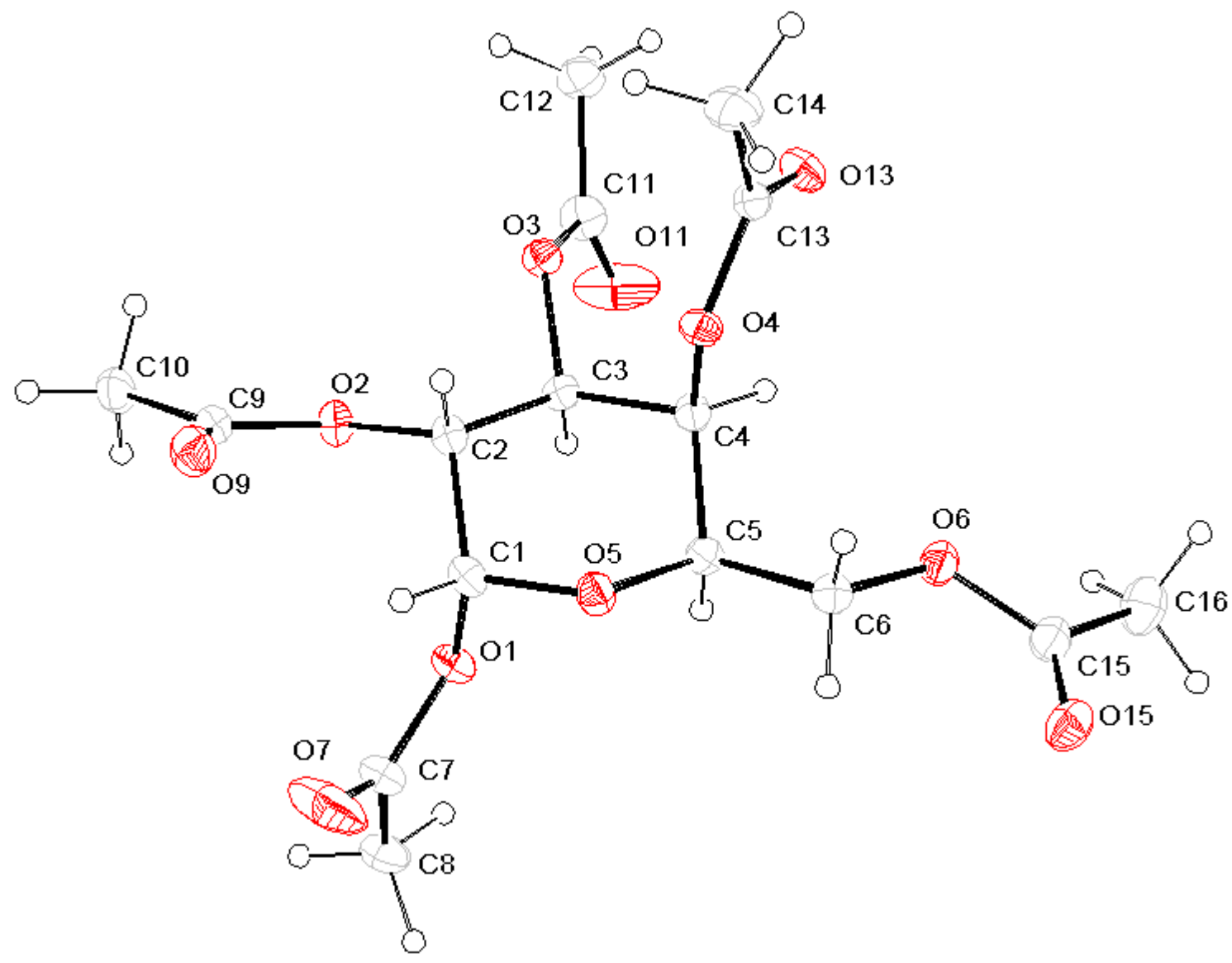

The structure of $\mathbf{5 a}$ derived from single-crystal X-ray analysis (ORTEP plot). The thermal displacement parameters are drawn at $20 \%$ probability. 
Table S9. Crystal data and structure refinement for $\mathbf{5 a}$.

Empirical formula

Formula weight

Temperature

Wavelength

Crystal system

Space group

Unit cell dimensions

Volume

Z

Density (calculated)

Absorption coefficient

$\mathrm{F}(000)$

Crystal size

Theta range for data collection

Index ranges

Reflections collected

Independent reflections

Completeness to theta $=25.68^{\circ}$

Absorption correction

Refinement method

Data / restraints / parameters

Goodness-of-fit on $\mathrm{F}^{2}$

Final $R$ indices $[\mathrm{I}>2 \sigma(\mathrm{I})]$

$\mathrm{R}$ indices (all data)

Absolute structure parameter

Largest diff. peak and hole
$\mathrm{C}_{16} \mathrm{H}_{22} \mathrm{O}_{11}$

390.34

173 (2) K

$0.71073 \AA$

orthorhombic

$P 2{ }_{1}{ }_{1} 2_{1}$, No. 19

$a=10.6486(3) \AA \quad \alpha=90^{\circ}$

$b=11.7152(3) \AA \quad \beta=90^{\circ}$

$c=15.0645(5) \AA \quad \gamma=90^{\circ}$

$1,879.30(9) \AA^{3}$

4

$1.380 \mathrm{Mg} / \mathrm{m}^{3}$

$0.118 \mathrm{~mm}^{-1}$

824

$0.30 \times 0.28 \times 0.16 \mathrm{~mm}$

$2.92^{\circ}-25.68^{\circ}$

$-12 \leq \mathrm{h} \leq 12,-14 \leq \mathrm{k} \leq 14,-18 \leq 1 \leq 18$

3,514

$3,514[\mathrm{R}(\mathrm{int})=0.0000]$

$99.1 \%$

none

full-matrix least-squares on $\mathrm{F}^{2}$

$3,514 / 0 / 272$

1.049

$\mathrm{R} 1=0.0442, \mathrm{wR} 2=0.0908$

$\mathrm{R} 1=0.0605, \mathrm{wR} 2=0.0991$

$0.2(12)$

0.201 and -0.178 e. $\AA^{-3}$ 
Table S10. Atomic coordinates $\left(\times 10^{4}\right)$ and equivalent isotropic displacement parameters $\left(A^{2} \times 10^{3}\right)$ for $5 \mathrm{a}$.

$\mathrm{U}(\mathrm{eq})$ is defined as one third of the trace of the orthogonalized Uij tensor.

\begin{tabular}{|c|c|c|c|c|}
\hline & $\mathrm{x}$ & $\mathrm{y}$ & z & $\mathrm{U}(\mathrm{eq})$ \\
\hline$O(1)$ & $1425(2)$ & $2042(1)$ & 5408 (1) & $32(1)$ \\
\hline$O(2)$ & $2253(2)$ & $3113(2)$ & $6933(1)$ & $30(1)$ \\
\hline$O(3)$ & $1410(2)$ & $5327(2)$ & $6722(1)$ & $26(1)$ \\
\hline$O(4)$ & $2275(2)$ & $5752(1)$ & $5027(1)$ & $26(1)$ \\
\hline$O(5)$ & $2295(2)$ & $3440(1)$ & $4494(1)$ & $29(1)$ \\
\hline$O(6)$ & $106(2)$ & $5256(2)$ & $3355(1)$ & $34(1)$ \\
\hline$O(7)$ & $2756(3)$ & $643(2)$ & $5021(2)$ & $82(1)$ \\
\hline$O(9)$ & $4223(2)$ & $2441(2)$ & $6807(1)$ & $40(1)$ \\
\hline$O(11)$ & $-649(2)$ & $5385(3)$ & $6977(2)$ & $80(1)$ \\
\hline$O(13)$ & $1230(2)$ & $7306(2)$ & $5518(1)$ & $35(1)$ \\
\hline$O(15)$ & $182(2)$ & $4865(2)$ & $1899(1)$ & $41(1)$ \\
\hline$C(1)$ & $2419(2)$ & $2866(2)$ & $5309(2)$ & $28(1)$ \\
\hline$C(2)$ & $2356(2)$ & $3693(2)$ & $6091(2)$ & $25(1)$ \\
\hline$C(3)$ & $1234(2)$ & $4476(2)$ & $6042(2)$ & $24(1)$ \\
\hline$C(4)$ & $1167(2)$ & $5050(2)$ & $5137(2)$ & $25(1)$ \\
\hline$C(5)$ & $1186(2)$ & $4147(2)$ & $4421(2)$ & $27(1)$ \\
\hline$C(6)$ & $1227(3)$ & $4583(2)$ & $3480(2)$ & $31(1)$ \\
\hline$C(7)$ & $1718(3)$ & $936(2)$ & $5232(2)$ & $42(1)$ \\
\hline$C(8)$ & $596(3)$ & $185(2)$ & $5305(2)$ & $48(1)$ \\
\hline$C(9)$ & $3288(3)$ & $2568(2)$ & $7247(2)$ & $31(1)$ \\
\hline$C(10)$ & $3085(3)$ & $2188(3)$ & $8178(2)$ & $39(1)$ \\
\hline$C(11)$ & $366(3)$ & $5770(3)$ & $7102(2)$ & $37(1)$ \\
\hline$C(12)$ & $668(3)$ & $6783(3)$ & $7660(2)$ & $39(1)$ \\
\hline$C(13)$ & $2194(2)$ & $6862(2)$ & $5301(2)$ & $28(1)$ \\
\hline$C(14)$ & $3458(2)$ & $7403(3)$ & $5289(2)$ & $44(1)$ \\
\hline$C(15)$ & $-346(3)$ & $5299(3)$ & $2517(2)$ & $34(1)$ \\
\hline$C(16)$ & $-1553(3)$ & $5942(3)$ & $2475(2)$ & 49 (1) \\
\hline
\end{tabular}


Table S11. Bond lengths [A] and angles [deg] for $5 \mathbf{a}$.

\begin{tabular}{|c|c|}
\hline $\mathrm{O}(1)-\mathrm{C}(7)$ & $1.358(3)$ \\
\hline $\mathrm{O}(1)-\mathrm{C}(1)$ & $1.441(3)$ \\
\hline $\mathrm{O}(2)-\mathrm{C}(9)$ & $1.359(3)$ \\
\hline $\mathrm{O}(2)-\mathrm{C}(2)$ & $1.443(3)$ \\
\hline$O(3)-C(11)$ & $1.354(3)$ \\
\hline$O(3)-C(3)$ & $1.440(3)$ \\
\hline $\mathrm{O}(4)-\mathrm{C}(13)$ & $1.367(3)$ \\
\hline $\mathrm{O}(4)-\mathrm{C}(4)$ & $1.447(3)$ \\
\hline $\mathrm{O}(5)-\mathrm{C}(1)$ & $1.406(3)$ \\
\hline$O(5)-C(5)$ & $1.446(3)$ \\
\hline $\mathrm{O}(6)-\mathrm{C}(15)$ & $1.352(3)$ \\
\hline $\mathrm{O}(6)-\mathrm{C}(6)$ & $1.443(3)$ \\
\hline $\mathrm{O}(7)-\mathrm{C}(7)$ & $1.201(4)$ \\
\hline $\mathrm{O}(9)-\mathrm{C}(9)$ & $1.206(3)$ \\
\hline $\mathrm{O}(11)-\mathrm{C}(11)$ & $1.186(4)$ \\
\hline $\mathrm{O}(13)-\mathrm{C}(13)$ & $1.197(3)$ \\
\hline $\mathrm{O}(15)-\mathrm{C}(15)$ & $1.200(3)$ \\
\hline$C(1)-C(2)$ & $1.527(4)$ \\
\hline $\mathrm{C}(1)-\mathrm{H}(1)$ & $0.99(2)$ \\
\hline$C(2)-C(3)$ & $1.509(3)$ \\
\hline $\mathrm{C}(2)-\mathrm{H}(2)$ & $0.92(2)$ \\
\hline$C(3)-C(4)$ & $1.522(3)$ \\
\hline $\mathrm{C}(3)-\mathrm{H}(3)$ & $1.01(2)$ \\
\hline$C(4)-C(5)$ & $1.512(4)$ \\
\hline $\mathrm{C}(4)-\mathrm{H}(4)$ & $0.98(2)$ \\
\hline$C(5)-C(6)$ & $1.508(4)$ \\
\hline $\mathrm{C}(5)-\mathrm{H}(5)$ & $1.00(3)$ \\
\hline $\mathrm{C}(6)-\mathrm{H}(6 \mathrm{~A})$ & $1.02(3)$ \\
\hline $\mathrm{C}(6)-\mathrm{H}(6 \mathrm{~B})$ & $0.93(3)$ \\
\hline$C(7)-C(8)$ & $1.487(4)$ \\
\hline $\mathrm{C}(8)-\mathrm{H}(8 \mathrm{~A})$ & 0.9800 \\
\hline $\mathrm{C}(8)-\mathrm{H}(8 \mathrm{~B})$ & 0.9800 \\
\hline $\mathrm{C}(8)-\mathrm{H}(8 \mathrm{C})$ & 0.9800 \\
\hline$C(9)-C(10)$ & $1.487(4)$ \\
\hline $\mathrm{C}(10)-\mathrm{H}(10 \mathrm{~A})$ & 0.9800 \\
\hline $\mathrm{C}(10)-\mathrm{H}(10 \mathrm{~B})$ & 0.9800 \\
\hline $\mathrm{C}(10)-\mathrm{H}(10 \mathrm{C})$ & 0.9800 \\
\hline$C(11)-C(12)$ & $1.490(4)$ \\
\hline $\mathrm{C}(12)-\mathrm{H}(12 \mathrm{~A})$ & 0.9800 \\
\hline $\mathrm{C}(12)-\mathrm{H}(12 \mathrm{~B})$ & 0.9800 \\
\hline $\mathrm{C}(12)-\mathrm{H}(12 \mathrm{C})$ & 0.9800 \\
\hline$C(13)-C(14)$ & $1.488(4)$ \\
\hline $\mathrm{C}(14)-\mathrm{H}(14 \mathrm{~A})$ & 0.9800 \\
\hline $\mathrm{C}(14)-\mathrm{H}(14 \mathrm{~B})$ & 0.9800 \\
\hline $\mathrm{C}(14)-\mathrm{H}(14 \mathrm{C})$ & 0.9800 \\
\hline$C(15)-C(16)$ & $1.491(4)$ \\
\hline $\mathrm{C}(16)-\mathrm{H}(16 \mathrm{~A})$ & 0.9800 \\
\hline $\mathrm{C}(16)-\mathrm{H}(16 \mathrm{~B})$ & 0.9800 \\
\hline $\mathrm{C}(16)-\mathrm{H}(16 \mathrm{C})$ & 0.9800 \\
\hline$C(7)-O(1)-C(1)$ & $116.7(2)$ \\
\hline$C(9)-O(2)-C(2)$ & $117.78(19)$ \\
\hline$C(11)-O(3)-C(3)$ & $117.3(2)$ \\
\hline$C(13)-O(4)-C(4)$ & $117.00(19)$ \\
\hline$C(1)-O(5)-C(5)$ & $114.69(19)$ \\
\hline
\end{tabular}




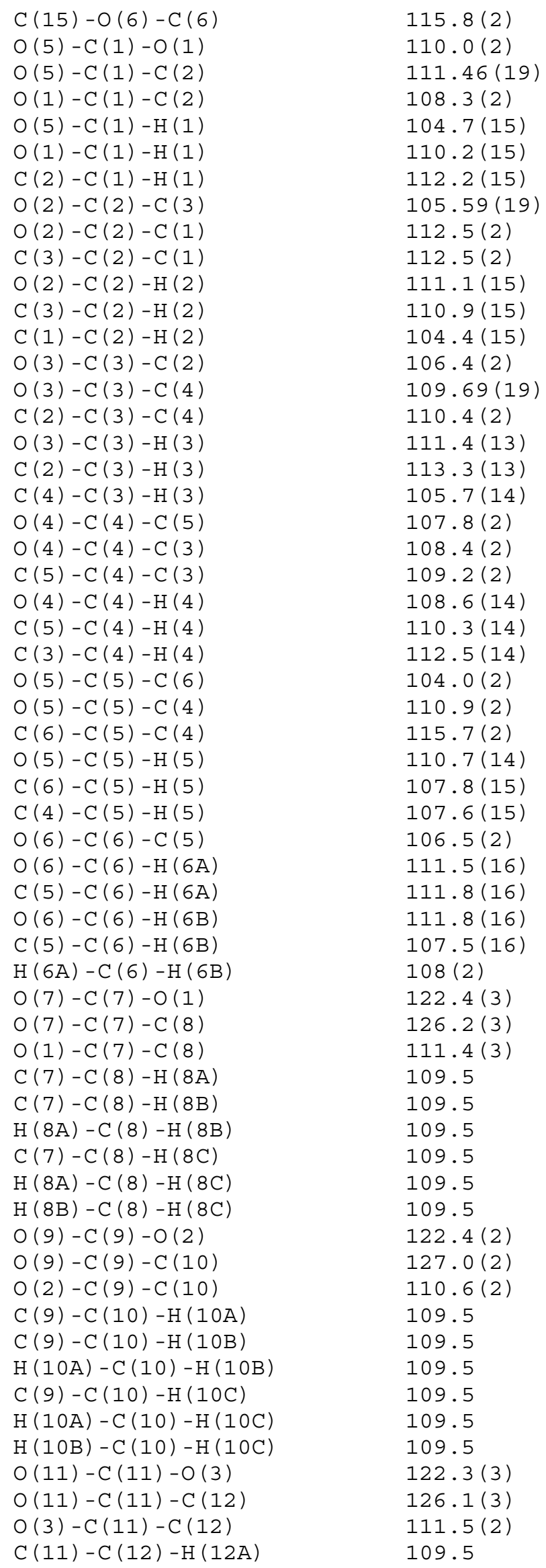




$\begin{array}{ll}\mathrm{C}(11)-\mathrm{C}(12)-\mathrm{H}(12 \mathrm{~B}) & 109.5 \\ \mathrm{H}(12 \mathrm{~A})-\mathrm{C}(12)-\mathrm{H}(12 \mathrm{~B}) & 109.5 \\ \mathrm{C}(11)-\mathrm{C}(12)-\mathrm{H}(12 \mathrm{C}) & 109.5 \\ \mathrm{H}(12 \mathrm{~A})-\mathrm{C}(12)-\mathrm{H}(12 \mathrm{C}) & 109.5 \\ \mathrm{H}(12 \mathrm{~B})-\mathrm{C}(12)-\mathrm{H}(12 \mathrm{C}) & 109.5 \\ \mathrm{O}(13)-\mathrm{C}(13)-\mathrm{O}(4) & 123.4(2) \\ \mathrm{O}(13)-\mathrm{C}(13)-\mathrm{C}(14) & 126.5(2) \\ \mathrm{O}(4)-\mathrm{C}(13)-\mathrm{C}(14) & 110.2(2) \\ \mathrm{C}(13)-\mathrm{C}(14)-\mathrm{H}(14 \mathrm{~A}) & 109.5 \\ \mathrm{C}(13)-\mathrm{C}(14)-\mathrm{H}(14 \mathrm{~B}) & 109.5 \\ \mathrm{H}(14 \mathrm{~A})-\mathrm{C}(14)-\mathrm{H}(14 \mathrm{~B}) & 109.5 \\ \mathrm{C}(13)-\mathrm{C}(14)-\mathrm{H}(14 \mathrm{C}) & 109.5 \\ \mathrm{H}(14 \mathrm{~A})-\mathrm{C}(14)-\mathrm{H}(14 \mathrm{C}) & 109.5 \\ \mathrm{H}(14 \mathrm{~B})-\mathrm{C}(14)-\mathrm{H}(14 \mathrm{C}) & 109.5 \\ \mathrm{O}(15)-\mathrm{C}(15)-\mathrm{O}(6) & 122.8(3) \\ \mathrm{O}(15)-\mathrm{C}(15)-\mathrm{C}(16) & 125.8(3) \\ \mathrm{O}(6)-\mathrm{C}(15)-\mathrm{C}(16) & 111.4(2) \\ \mathrm{C}(15)-\mathrm{C}(16)-\mathrm{H}(16 \mathrm{~A}) & 109.5 \\ \mathrm{C}(15)-\mathrm{C}(16)-\mathrm{H}(16 \mathrm{~B}) & 109.5 \\ \mathrm{H}(16 \mathrm{~A})-\mathrm{C}(16)-\mathrm{H}(16 \mathrm{~B}) & 109.5 \\ \mathrm{C}(15)-\mathrm{C}(16)-\mathrm{H}(16 \mathrm{C}) & 109.5 \\ \mathrm{H}(16 \mathrm{~A})-\mathrm{C}(16)-\mathrm{H}(16 \mathrm{C}) & 109.5 \\ \mathrm{H}(16 \mathrm{~B})-\mathrm{C}(16)-\mathrm{H}(16 \mathrm{C}) & 109.5 \\ \end{array}$

Symmetry transformations used to generate equivalent atoms: 
Table S12. Anisotropic displacement parameters $\left(A^{2} \times 10^{3}\right)$ for $5 a$. The anisotropic displacement factor exponent takes the form:

$-2 \mathrm{pi}^{2}\left[\mathrm{~h}^{2} \mathrm{a} *^{2} \mathrm{U} 11+\ldots+2 \mathrm{hk} \mathrm{k} * \mathrm{~b} * \mathrm{U} 12\right]$

\begin{tabular}{|c|c|c|c|c|c|c|}
\hline & $\mathrm{U} 11$ & U22 & U33 & U2 3 & U13 & U12 \\
\hline$O(1)$ & $36(1)$ & $21(1)$ & $39(1)$ & $-1(1)$ & $3(1)$ & $-1(1)$ \\
\hline$O(2)$ & $29(1)$ & $32(1)$ & $29(1)$ & $7(1)$ & $0(1)$ & $5(1)$ \\
\hline$O(3)$ & $24(1)$ & $29(1)$ & $26(1)$ & $-4(1)$ & $2(1)$ & $3(1)$ \\
\hline$O(4)$ & $24(1)$ & $23(1)$ & $32(1)$ & $-1(1)$ & $2(1)$ & $-4(1)$ \\
\hline$O(5)$ & $31(1)$ & $28(1)$ & $28(1)$ & $2(1)$ & $2(1)$ & $5(1)$ \\
\hline$O(6)$ & $37(1)$ & $36(1)$ & $29(1)$ & $0(1)$ & $-6(1)$ & $6(1)$ \\
\hline$O(7)$ & $60(2)$ & $39(1)$ & $147(3)$ & $-27(2)$ & $6(2)$ & $10(1)$ \\
\hline$O(9)$ & $35(1)$ & $43(1)$ & $43(1)$ & $-2(1)$ & $-2(1)$ & $12(1)$ \\
\hline$O(11)$ & $28(1)$ & $88(2)$ & $123(2)$ & $-60(2)$ & $21(1)$ & $-11(1)$ \\
\hline$O(13)$ & $35(1)$ & $25(1)$ & $46(1)$ & $-1(1)$ & $4(1)$ & $5(1)$ \\
\hline$O(15)$ & $42(1)$ & $51(1)$ & 29 (1) & $3(1)$ & $0(1)$ & $-7(1)$ \\
\hline$C(1)$ & $27(1)$ & $23(1)$ & $35(1)$ & $3(1)$ & $2(1)$ & $1(1)$ \\
\hline$C(2)$ & $23(1)$ & $26(1)$ & $28(1)$ & $4(1)$ & $2(1)$ & $1(1)$ \\
\hline$C(3)$ & $22(1)$ & $27(1)$ & $25(1)$ & $-2(1)$ & $1(1)$ & $0(1)$ \\
\hline $\mathrm{C}(4)$ & $21(1)$ & $25(1)$ & $29(1)$ & $4(1)$ & $-2(1)$ & $-3(1)$ \\
\hline C (5) & $26(1)$ & $26(1)$ & $28(1)$ & $0(1)$ & $-2(1)$ & $0(1)$ \\
\hline$C(6)$ & $33(2)$ & $29(1)$ & $30(1)$ & $-2(1)$ & $-2(1)$ & $2(1)$ \\
\hline $\mathrm{C}(7)$ & $55(2)$ & $25(1)$ & $46(2)$ & $-6(1)$ & $-6(2)$ & $6(1)$ \\
\hline$C(8)$ & $68(2)$ & $24(1)$ & $52(2)$ & $-4(2)$ & $-6(2)$ & $-7(1)$ \\
\hline$C(9)$ & $35(2)$ & $24(1)$ & $33(1)$ & $-2(1)$ & $-6(1)$ & $5(1)$ \\
\hline$C(10)$ & $52(2)$ & $36(2)$ & $30(2)$ & $2(1)$ & $-3(1)$ & $9(1)$ \\
\hline$C(11)$ & $30(2)$ & $42(2)$ & $39(2)$ & $-6(1)$ & $8(1)$ & $6(1)$ \\
\hline$C(12)$ & $41(2)$ & $39(2)$ & $37(2)$ & $-10(1)$ & $6(1)$ & $6(1)$ \\
\hline$C(13)$ & $34(1)$ & $25(1)$ & $24(1)$ & $2(1)$ & $-2(1)$ & $-2(1)$ \\
\hline$C(14)$ & $35(2)$ & $37(2)$ & $59(2)$ & $-5(2)$ & $-2(1)$ & $-13(1)$ \\
\hline$C(15)$ & $36(2)$ & $36(2)$ & $32(2)$ & $6(1)$ & $-5(1)$ & $-10(1)$ \\
\hline$C(16)$ & $36(2)$ & $63(2)$ & $48(2)$ & $7(2)$ & $-8(1)$ & $2(2)$ \\
\hline
\end{tabular}


Table S13. Hydrogen coordinates $\left(\times 10^{4}\right)$ and isotropic displacement parameters $\left(A^{2} \times 10^{3}\right)$ for $5 \mathrm{a}$.

\begin{tabular}{|c|c|c|c|c|}
\hline & $\mathrm{x}$ & $\mathrm{Y}$ & $\mathrm{z}$ & $\mathrm{U}(\mathrm{eq})$ \\
\hline $\mathrm{H}(8 \mathrm{~A})$ & 496 & -252 & 4755 & 72 \\
\hline $\mathrm{H}(8 \mathrm{~B})$ & -153 & 654 & 5405 & 72 \\
\hline $\mathrm{H}(8 \mathrm{C})$ & 707 & -341 & 5805 & 72 \\
\hline $\mathrm{H}(10 \mathrm{~A})$ & 3841 & 1800 & 8395 & 59 \\
\hline $\mathrm{H}(10 \mathrm{~B})$ & 2371 & 1661 & 8201 & 59 \\
\hline $\mathrm{H}(10 \mathrm{C})$ & 2910 & 2853 & 8553 & 59 \\
\hline $\mathrm{H}(12 \mathrm{~A})$ & 868 & 7434 & 7277 & 58 \\
\hline $\mathrm{H}(12 \mathrm{~B})$ & 1391 & 6610 & 8039 & 58 \\
\hline $\mathrm{H}(12 \mathrm{C})$ & -58 & 6972 & 8032 & 58 \\
\hline $\mathrm{H}(14 \mathrm{~A})$ & 3368 & 8233 & 5341 & 65 \\
\hline $\mathrm{H}(14 \mathrm{~B})$ & 3882 & 7219 & 4729 & 65 \\
\hline $\mathrm{H}(14 \mathrm{C})$ & 3957 & 7115 & 5787 & 65 \\
\hline $\mathrm{H}(16 \mathrm{~A})$ & -1378 & 6758 & 2406 & 73 \\
\hline $\mathrm{H}(16 \mathrm{~B})$ & -2026 & 5817 & 3025 & 73 \\
\hline $\mathrm{H}(16 \mathrm{C})$ & -2047 & 5672 & 1968 & 73 \\
\hline $\mathrm{H}(1)$ & $3240(20)$ & $2470(20)$ & $5271(17)$ & $25(6)$ \\
\hline $\mathrm{H}(2)$ & $3090(20)$ & $4100(20)$ & $6057(16)$ & $15(6)$ \\
\hline $\mathrm{H}(3)$ & $410(20)$ & $4060(20)$ & $6117(16)$ & $18(6)$ \\
\hline $\mathrm{H}(4)$ & $420(20)$ & $5540(20)$ & $5073(16)$ & $21(6)$ \\
\hline $\mathrm{H}(5)$ & $410(20)$ & $3680(20)$ & $4487(17)$ & $27(7)$ \\
\hline $\mathrm{H}(6 \mathrm{~A})$ & $2020(30)$ & $5050(30)$ & $3360(19)$ & $40(8)$ \\
\hline $\mathrm{H}(6 \mathrm{~B})$ & $1240(20)$ & $3950(20)$ & 3107 (18) & $25(7)$ \\
\hline
\end{tabular}


Table s14. Torsion angles [deg] for 5a.

\begin{tabular}{|c|c|}
\hline$C(5)-O(5)-C(1)-O(1)$ & $65.7(2)$ \\
\hline$C(5)-O(5)-C(1)-C(2)$ & $-54.3(3)$ \\
\hline$C(7)-O(1)-C(1)-O(5)$ & $100.6(3)$ \\
\hline$C(7)-O(1)-C(1)-C(2)$ & $-137.4(2)$ \\
\hline$C(9)-O(2)-C(2)-C(3)$ & $-164.3(2)$ \\
\hline$C(9)-O(2)-C(2)-C(1)$ & $72.6(3)$ \\
\hline$O(5)-C(1)-C(2)-O(2)$ & $169.5(2)$ \\
\hline$O(1)-C(1)-C(2)-O(2)$ & $48.4(3)$ \\
\hline$O(5)-C(1)-C(2)-C(3)$ & $50.3(3)$ \\
\hline$O(1)-C(1)-C(2)-C(3)$ & $-70.7(3)$ \\
\hline$C(11)-O(3)-C(3)-C(2)$ & $-149.4(2)$ \\
\hline$C(11)-O(3)-C(3)-C(4)$ & $91.1(3)$ \\
\hline$O(2)-C(2)-C(3)-O(3)$ & $66.7(2)$ \\
\hline$C(1)-C(2)-C(3)-O(3)$ & $-170.2(2)$ \\
\hline$O(2)-C(2)-C(3)-C(4)$ & $-174.28(19)$ \\
\hline$C(1)-C(2)-C(3)-C(4)$ & $-51.2(3)$ \\
\hline$C(13)-O(4)-C(4)-C(5)$ & $151.2(2)$ \\
\hline$C(13)-O(4)-C(4)-C(3)$ & $-90.7(2)$ \\
\hline$O(3)-C(3)-C(4)-O(4)$ & $54.1(3)$ \\
\hline$C(2)-C(3)-C(4)-O(4)$ & $-62.9(2)$ \\
\hline$O(3)-C(3)-C(4)-C(5)$ & $171.24(19)$ \\
\hline$C(2)-C(3)-C(4)-C(5)$ & $54.3(3)$ \\
\hline$C(1)-O(5)-C(5)-C(6)$ & $-175.9(2)$ \\
\hline$C(1)-O(5)-C(5)-C(4)$ & $59.1(3)$ \\
\hline$O(4)-C(4)-C(5)-O(5)$ & $60.3(2)$ \\
\hline$C(3)-C(4)-C(5)-O(5)$ & $-57.3(3)$ \\
\hline$O(4)-C(4)-C(5)-C(6)$ & $-57.9(3)$ \\
\hline$C(3)-C(4)-C(5)-C(6)$ & $-175.4(2)$ \\
\hline$C(15)-O(6)-C(6)-C(5)$ & $-151.3(2)$ \\
\hline$O(5)-C(5)-C(6)-O(6)$ & $177.44(19)$ \\
\hline$C(4)-C(5)-C(6)-O(6)$ & $-60.6(3)$ \\
\hline$C(1)-O(1)-C(7)-O(7)$ & $1.4(5)$ \\
\hline$C(1)-O(1)-C(7)-C(8)$ & $-176.6(2)$ \\
\hline$C(2)-O(2)-C(9)-O(9)$ & $-8.8(4)$ \\
\hline$C(2)-O(2)-C(9)-C(10)$ & $170.5(2)$ \\
\hline$C(3)-O(3)-C(11)-O(11)$ & $9.7(4)$ \\
\hline$C(3)-O(3)-C(11)-C(12)$ & $-169.2(2)$ \\
\hline$C(4)-O(4)-C(13)-O(13)$ & $-9.5(3)$ \\
\hline$C(4)-O(4)-C(13)-C(14)$ & $170.3(2)$ \\
\hline$C(6)-O(6)-C(15)-O(15)$ & $-4.2(4)$ \\
\hline$C(6)-O(6)-C(15)-C(16)$ & $175.8(2)$ \\
\hline
\end{tabular}

Symmetry transformations used to generate equivalent atoms: 
Table s15. XYZ file for 5a.

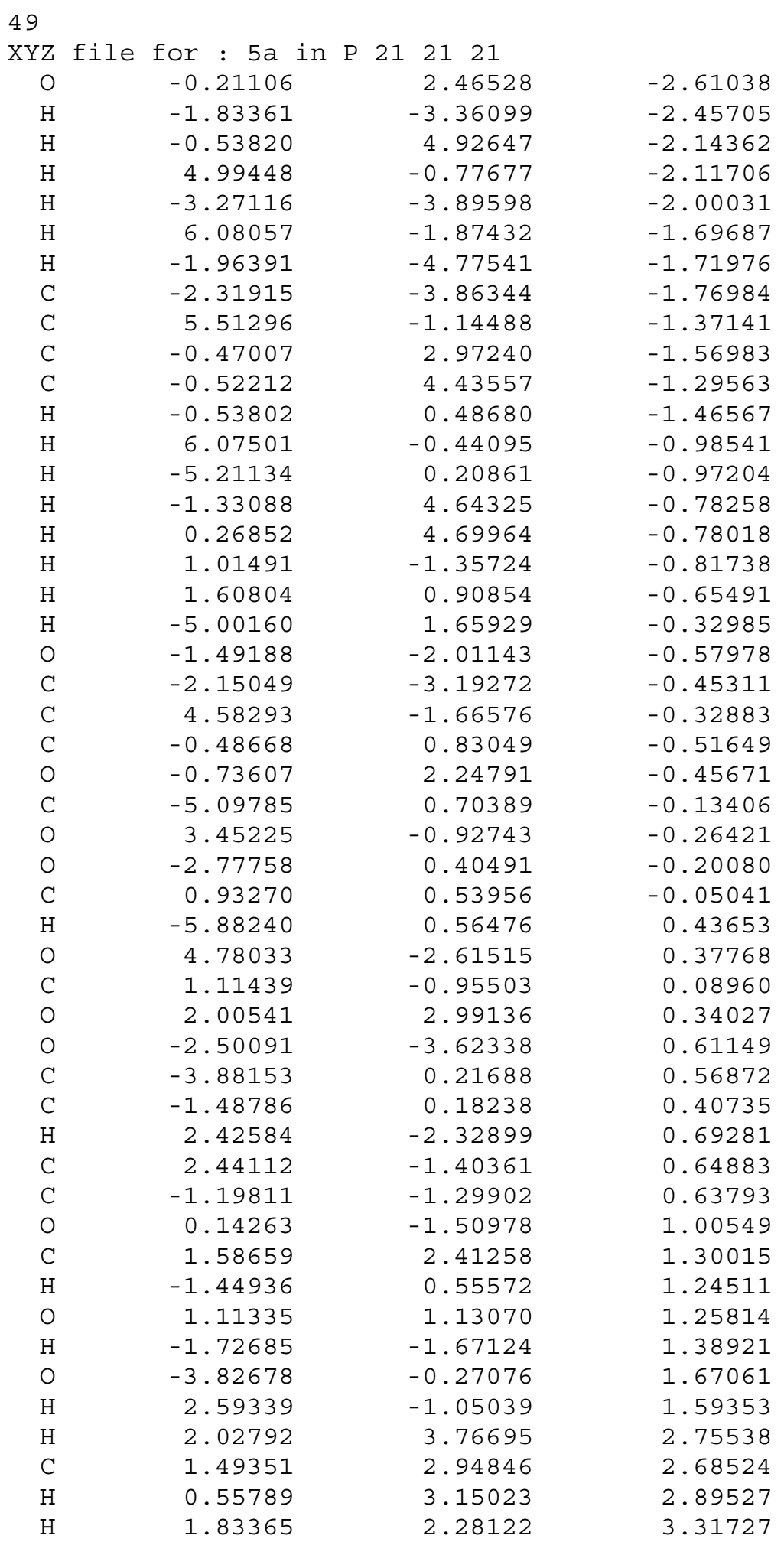




\section{Computational data}

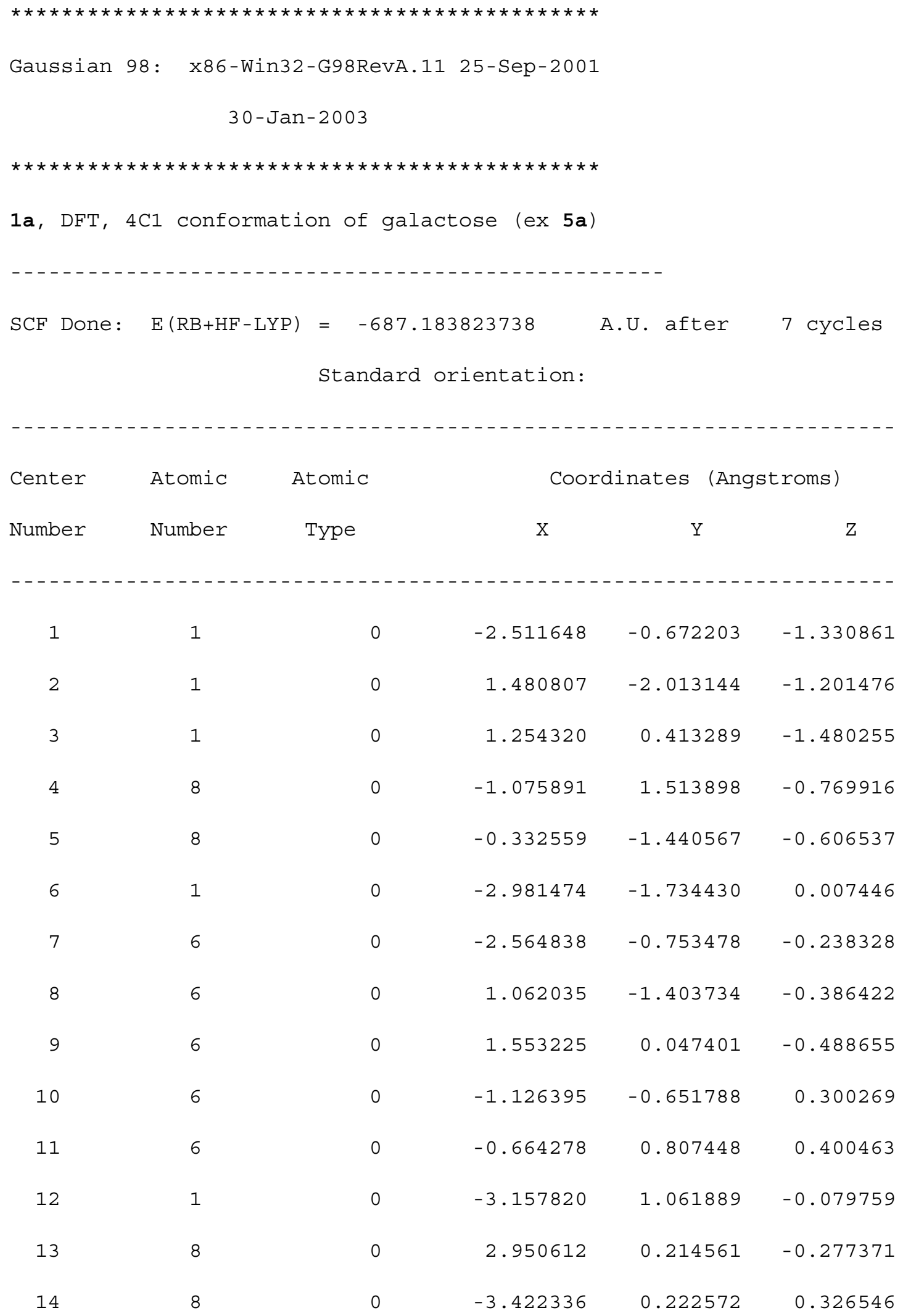




$\begin{array}{cccccc}15 & 1 & 0 & 1.123673 & -2.829710 & 0.911674 \\ 16 & 8 & 0 & 1.208632 & 2.272864 & 0.414619 \\ 17 & 6 & 0 & 0.854484 & 0.900130 & 0.564371 \\ 19 & 8 & 0 & 1.436548 & -1.915430 & 0.869180 \\ 20 & 1 & 0 & -1.143817 & 1.246110 & 1.287238 \\ 21 & 1 & 0 & -1.059660 & -1.081945 & 1.308277 \\ 22 & 1 & 0 & 2.166296 & 2.284598 & 0.268024 \\ 23 & 1 & 0 & 1.140154 & 0.529179 & 1.557165 \\ 24 & 1 & 0 & 3.426210 & -0.147906 & -1.035612 \\ --1 & 1 & 0 & -0.542499 & 2.325216 & -0.774217\end{array}$

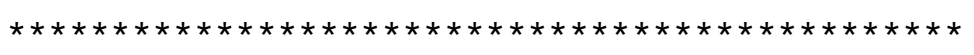

Gaussian 98: x86-Win32-G98RevA.11 25-Sep-2001

21-Jun-2002

1a, DFT, 4C1 conformation of galactose (ex 3 )

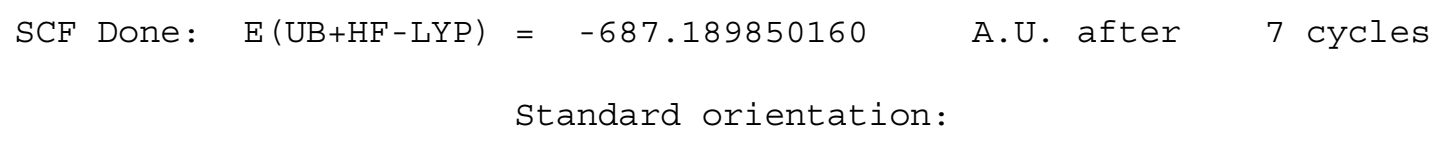

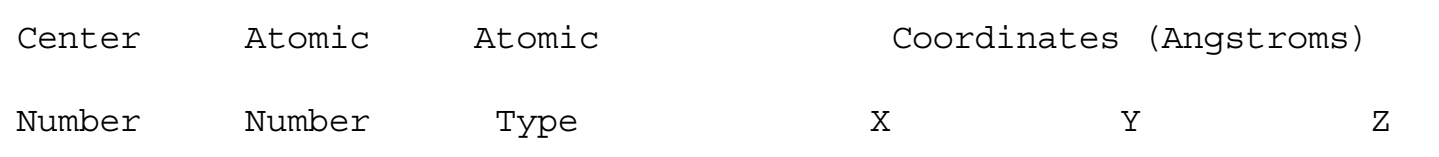

$\begin{array}{llllll}1 & 8 & 0 & -0.278589 & 2.017198 & -0.784886 \\ 2 & 6 & 0 & -0.191457 & 1.267216 & 0.416036 \\ 3 & 1 & 0 & 0.537434 & 2.541222 & -0.815314 \\ 4 & 1 & 0 & -0.411980 & 1.904947 & 1.288844\end{array}$




\begin{tabular}{|c|c|c|c|c|c|}
\hline 5 & 6 & 0 & -1.214622 & 0.129444 & 0.397846 \\
\hline 6 & 6 & 0 & 1.218320 & 0.687420 & 0.570725 \\
\hline 7 & 6 & 0 & 1.478659 & -0.364211 & -0.511595 \\
\hline 8 & 6 & 0 & -2.629922 & 0.577217 & 0.047663 \\
\hline 9 & 1 & 0 & -1.223332 & -0.338041 & 1.391626 \\
\hline 10 & 8 & 0 & -0.889935 & -0.883666 & -0.582736 \\
\hline 11 & 8 & 0 & 2.143502 & 1.761350 & 0.442491 \\
\hline 12 & 1 & 0 & 1.306929 & 0.213189 & 1.559816 \\
\hline 13 & 8 & 0 & 2.785846 & -0.909566 & -0.382553 \\
\hline 14 & 1 & 0 & 1.436287 & 0.129417 & -1.485909 \\
\hline 15 & 6 & 0 & 0.395291 & -1.445122 & -0.481686 \\
\hline 16 & 1 & 0 & -2.969817 & 1.314731 & 0.781566 \\
\hline 17 & 1 & 0 & -2.616722 & 1.057878 & -0.938848 \\
\hline 18 & 8 & 0 & -3.538893 & -0.510523 & 0.098851 \\
\hline 19 & 1 & 0 & 2.999891 & 1.352711 & 0.243205 \\
\hline 20 & 1 & 0 & 0.486527 & -2.109553 & -1.349594 \\
\hline 21 & 8 & 0 & 0.597630 & -2.166019 & 0.724117 \\
\hline 22 & 1 & 0 & -3.181768 & -1.174991 & -0.506970 \\
\hline 23 & 1 & 0 & 2.737765 & -1.544073 & 0.349724 \\
\hline 24 & 1 & 0 & 0.004671 & -2.929404 & 0.725657 \\
\hline
\end{tabular}

Gaussian 98: x86-Win32-G98RevA.11 25-Sep-2001 19-Jun-2002

1a, DFT, OS2 skew-boat conformation of galactose 


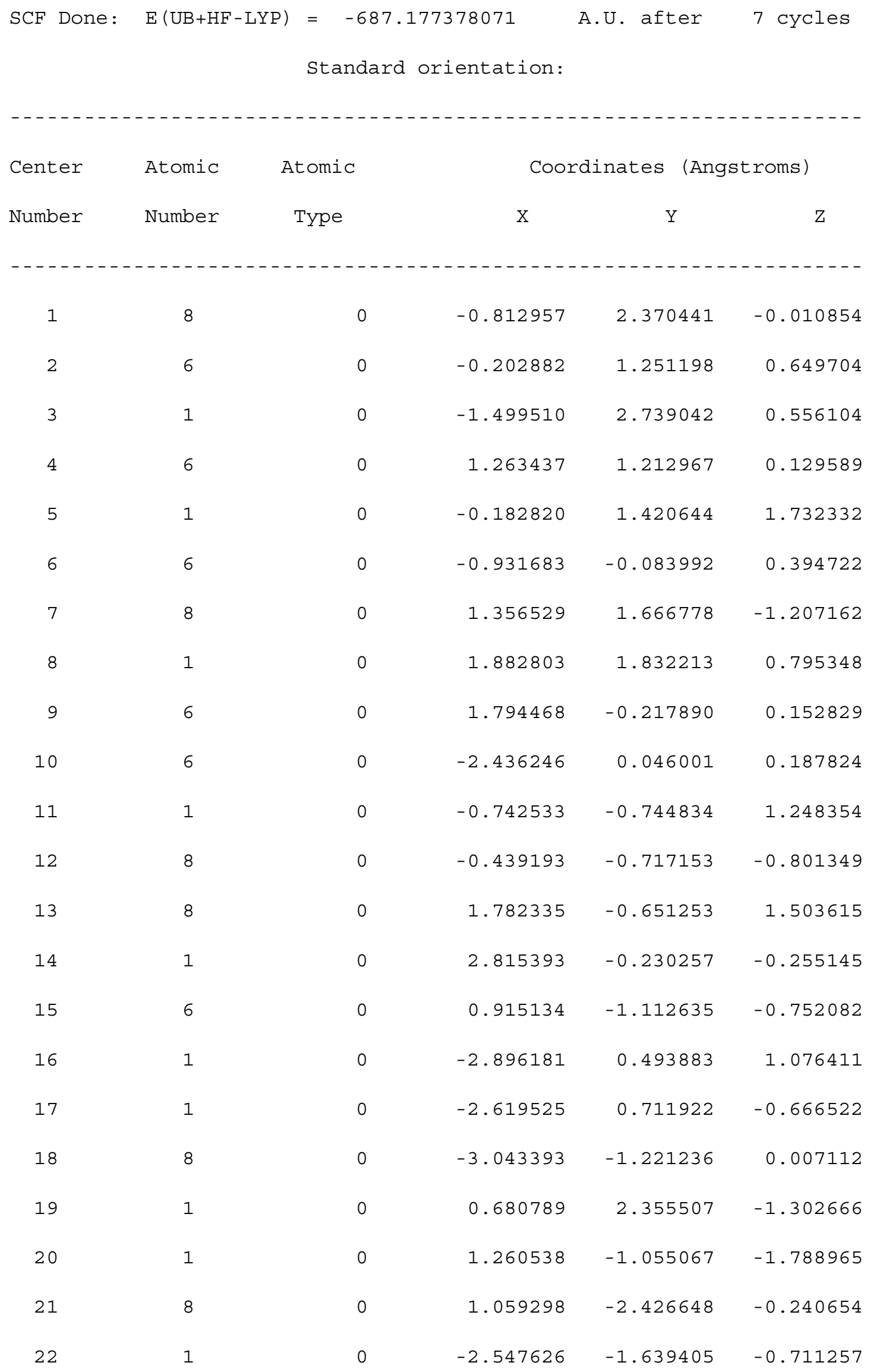




$\begin{array}{llllll}23 & 1 & 0 & 1.749535 & -1.620030 & 1.471179 \\ 24 & 1 & 0 & 0.464821 & -3.004938 & -0.736349\end{array}$

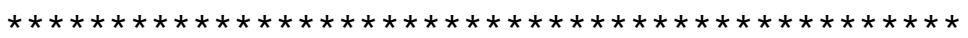

Gaussian 98: x86-Win32-G98RevA.11 25-Sep-2001

$06-$ Jun -2002
\end{abstract}

3, X-ray, unopt. Os2 skew-boat conf. of the isoprop. deriv. of galactose

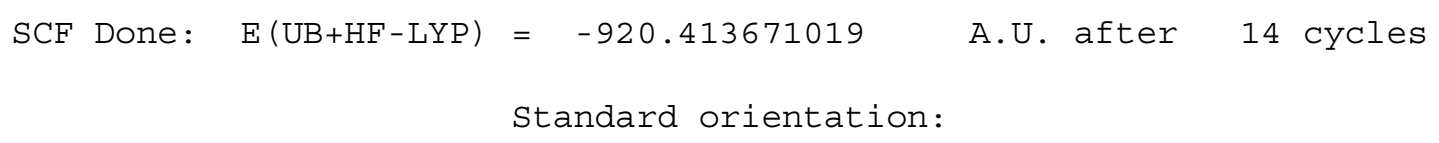

\begin{tabular}{|c|c|c|c|c|c|}
\hline \multirow{2}{*}{$\begin{array}{l}\text { Center } \\
\text { Number }\end{array}$} & \multirow{2}{*}{$\begin{array}{l}\text { Atomic } \\
\text { Number }\end{array}$} & \multirow{2}{*}{$\begin{array}{l}\text { Atomic } \\
\text { Type }\end{array}$} & \multicolumn{3}{|c|}{ Coordinates (Angstroms) } \\
\hline & & & $\mathrm{X}$ & $\mathrm{Y}$ & $\mathrm{Z}$ \\
\hline \multicolumn{6}{|c|}{ - - - - - - - - - - - - - - - - - - - - - - - - - - - - - - - - - - - - - - - - - - - - - - - - - - - - - - - - - - - - - - - - } \\
\hline 1 & 1 & 0 & -4.374364 & -0.895283 & -1.265219 \\
\hline 2 & 6 & 0 & -3.567270 & -1.383321 & -1.084180 \\
\hline 3 & 1 & 0 & -3.785896 & -2.287258 & -0.845948 \\
\hline 4 & 1 & 0 & -3.011650 & -1.384187 & -1.867191 \\
\hline 5 & 6 & 0 & -2.837503 & -0.735224 & 0.041872 \\
\hline 6 & 6 & 0 & -3.712081 & -0.583284 & 1.273337 \\
\hline 7 & 8 & 0 & -2.341006 & 0.536928 & -0.355841 \\
\hline 8 & 8 & 0 & -1.682108 & -1.456819 & 0.411969 \\
\hline 9 & 1 & 0 & -4.614728 & -0.828348 & 1.056227 \\
\hline 10 & 1 & 0 & -3.690624 & 0.328987 & 1.570570 \\
\hline 11 & 1 & 0 & -3.385064 & -1.155635 & 1.970712 \\
\hline 12 & 6 & 0 & -1.037936 & 0.357214 & -0.915926 \\
\hline
\end{tabular}




\begin{tabular}{|c|c|c|c|c|c|}
\hline 13 & 6 & 0 & -0.672695 & -1.073785 & -0.512799 \\
\hline 14 & 1 & 0 & -1.071244 & 0.440044 & -1.892161 \\
\hline 15 & 1 & 0 & -0.702887 & -1.658341 & -1.299081 \\
\hline 16 & 6 & 0 & -0.078489 & 1.379791 & -0.332296 \\
\hline 17 & 6 & 0 & 0.647270 & -1.229166 & 0.188529 \\
\hline 18 & 6 & 0 & -0.638867 & 2.780223 & -0.309939 \\
\hline 19 & 1 & 0 & 0.751177 & 1.375218 & -0.852731 \\
\hline 20 & 8 & 0 & 0.222748 & 1.051198 & 1.031066 \\
\hline 21 & 8 & 0 & 1.688370 & -1.150993 & -0.801805 \\
\hline 22 & 1 & 0 & 0.683261 & -2.094200 & 0.645992 \\
\hline 23 & 6 & 0 & 1.007667 & -0.101259 & 1.167289 \\
\hline 24 & 1 & 0 & -0.993456 & 3.004964 & -1.184177 \\
\hline 25 & 1 & 0 & -1.364269 & 2.831942 & 0.332935 \\
\hline 26 & 8 & 0 & 0.396499 & 3.714546 & 0.050055 \\
\hline 27 & 1 & 0 & 0.945442 & -0.433046 & 2.087275 \\
\hline 28 & 8 & 0 & 2.363229 & 0.213259 & 0.861943 \\
\hline 29 & 6 & 0 & 2.857608 & -0.688320 & -0.127325 \\
\hline 30 & 6 & 0 & 3.724894 & 0.050054 & -1.095358 \\
\hline 31 & 6 & 0 & 3.592822 & -1.836684 & 0.530892 \\
\hline 32 & 1 & 0 & 0.744646 & 3.488383 & 0.916139 \\
\hline 33 & 1 & 0 & 3.219398 & 0.757114 & -1.502189 \\
\hline 34 & 1 & 0 & 4.032422 & -0.554815 & -1.774388 \\
\hline 35 & 1 & 0 & 4.478695 & 0.420727 & -0.631110 \\
\hline 36 & 1 & 0 & 3.804277 & -2.501207 & -0.128558 \\
\hline 37 & 1 & 0 & 3.036801 & -2.225769 & 1.210403 \\
\hline 38 & 1 & 0 & 4.403696 & -1.511667 & 0.928829 \\
\hline
\end{tabular}




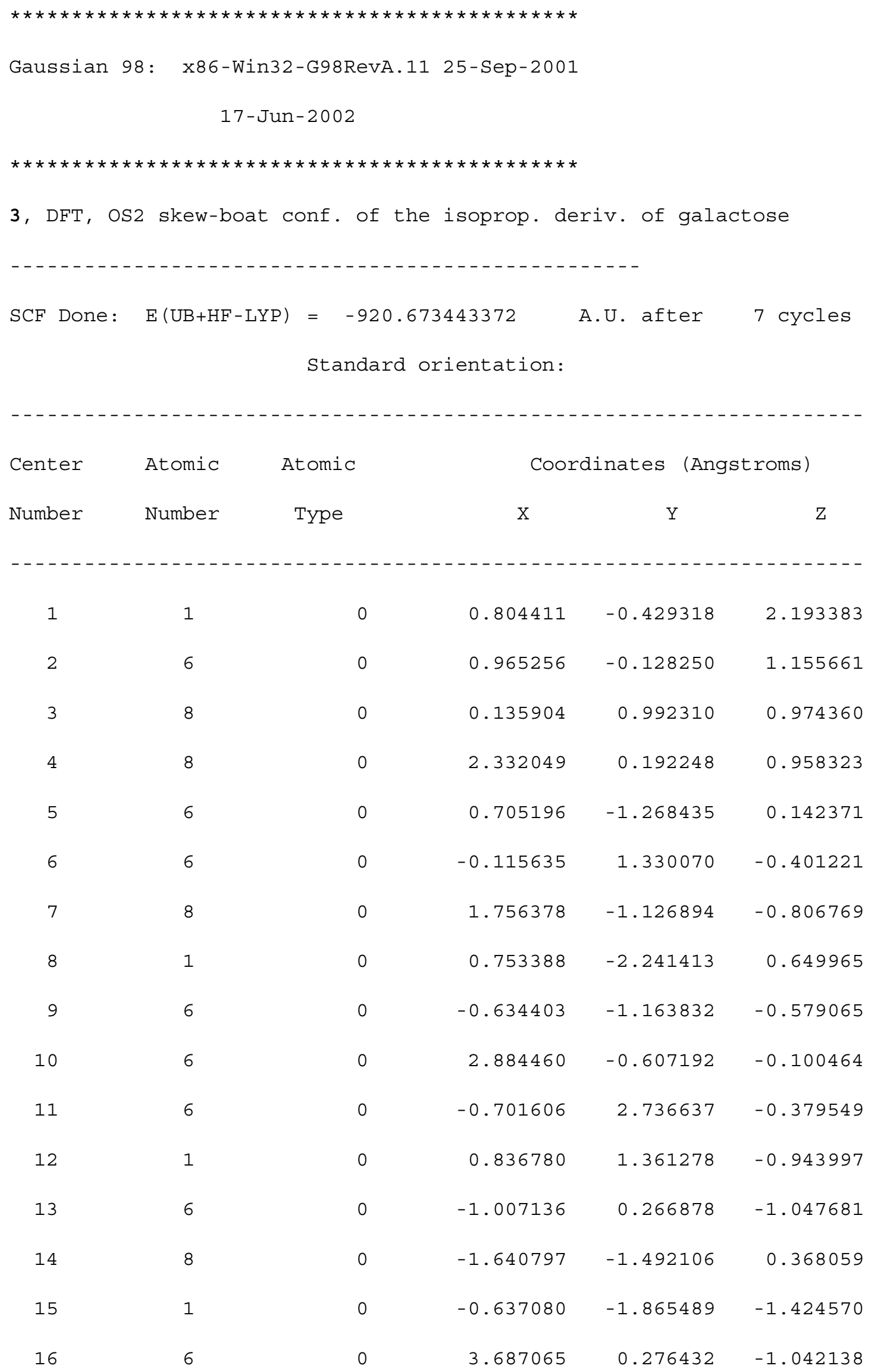




\begin{tabular}{|c|c|c|c|c|c|}
\hline 17 & 6 & 0 & 3.721235 & -1.740033 & 0.499705 \\
\hline 18 & 8 & 0 & 0.227683 & 3.659447 & 0.161463 \\
\hline 19 & 1 & 0 & -1.645157 & 2.721526 & 0.181502 \\
\hline 20 & 1 & 0 & -0.922877 & 3.060247 & -1.401984 \\
\hline 21 & 1 & 0 & -0.936310 & 0.358823 & -2.138611 \\
\hline 22 & 8 & 0 & -2.370421 & 0.415910 & -0.658412 \\
\hline 23 & 1 & 0 & 3.062735 & 1.085640 & -1.425928 \\
\hline 24 & 1 & 0 & 4.061645 & -0.313492 & -1.882751 \\
\hline 25 & 1 & 0 & 4.537946 & 0.711303 & -0.512097 \\
\hline 26 & 1 & 0 & 4.147606 & -2.359593 & -0.294021 \\
\hline 27 & 1 & 0 & 3.110560 & -2.375308 & 1.146997 \\
\hline 28 & 1 & 0 & 4.535073 & -1.325618 & 1.100459 \\
\hline 29 & 6 & 0 & -2.812863 & -0.730320 & 0.078661 \\
\hline 30 & 6 & 0 & -3.418164 & -0.274456 & 1.398625 \\
\hline 31 & 6 & 0 & -3.777387 & -1.542644 & -0.786588 \\
\hline 32 & 1 & 0 & 0.528279 & 3.267253 & 0.994407 \\
\hline 33 & 1 & 0 & -2.674425 & 0.305981 & 1.948080 \\
\hline 34 & 1 & 0 & -3.717778 & -1.137980 & 1.998734 \\
\hline 35 & 1 & 0 & -4.297838 & 0.348164 & 1.215707 \\
\hline 36 & 1 & 0 & -4.120234 & -2.429571 & -0.246569 \\
\hline 37 & 1 & 0 & -3.283530 & -1.863383 & -1.708246 \\
\hline 38 & 1 & 0 & -4.645664 & -0.935489 & -1.056562 \\
\hline
\end{tabular}

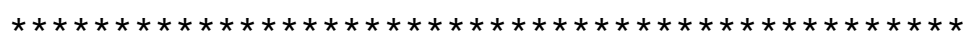

Gaussian 98: x86-Win32-G98RevA.11 25-Sep-2001

22 - Jan- 2003 
5a, X-ray, unopt. 4C1 conformation of the galactose pentaacetate

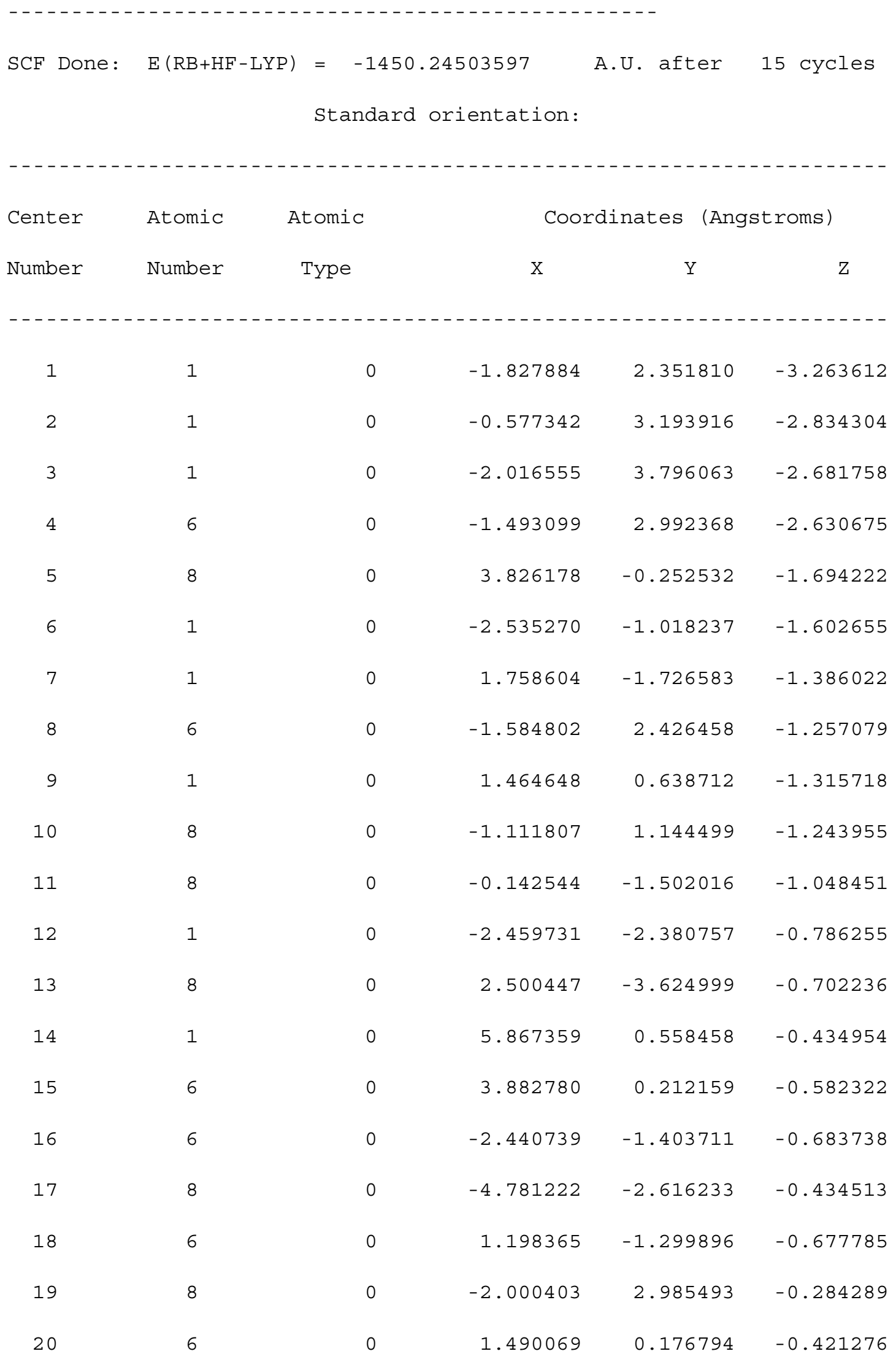




\begin{tabular}{|c|c|c|c|c|c|}
\hline 21 & 6 & 0 & 5.099585 & 0.683321 & 0.127320 \\
\hline 22 & 6 & 0 & -1.112250 & -0.965626 & -0.118045 \\
\hline 23 & 1 & 0 & 5.006759 & 1.615155 & 0.339134 \\
\hline 24 & 6 & 0 & -0.928706 & 0.524955 & 0.052951 \\
\hline 25 & 6 & 0 & -4.580014 & -1.682461 & 0.291871 \\
\hline 26 & 8 & 0 & 2.781469 & 0.383846 & 0.192647 \\
\hline 27 & 8 & 0 & -3.450519 & -0.943167 & 0.240151 \\
\hline 28 & 6 & 0 & 2.150924 & -3.217149 & 0.370268 \\
\hline 29 & 8 & 0 & 0.741544 & 2.223703 & 0.491429 \\
\hline 30 & 6 & 0 & 0.491461 & 0.805977 & 0.521140 \\
\hline 31 & 8 & 0 & 1.494179 & -2.037549 & 0.523395 \\
\hline 32 & 1 & 0 & 1. 322232 & 4.605669 & 0.875415 \\
\hline 33 & 1 & 0 & -0.244721 & 4.661961 & 0.877711 \\
\hline 34 & 1 & 0 & -1.646293 & 0.907037 & 0.605529 \\
\hline 35 & 1 & 0 & -6.060666 & -0.484488 & 0.984141 \\
\hline 36 & 1 & 0 & 5.211804 & 0.181086 & 0.937724 \\
\hline 37 & 1 & 0 & -0.996679 & -1.339435 & 0.816078 \\
\hline 38 & 6 & 0 & -5.509497 & -1.181743 & 1.347474 \\
\hline 39 & 6 & 0 & 0.531061 & 4.392782 & 1.375625 \\
\hline 40 & 1 & 0 & -6.065470 & -1.902820 & 1.651494 \\
\hline 41 & 1 & 0 & 1.973116 & -4.807284 & 1.604481 \\
\hline 42 & 6 & 0 & 0.478371 & 2.925347 & 1.620947 \\
\hline 43 & 1 & 0 & 0.584073 & 0.416623 & 1.481988 \\
\hline 44 & 6 & 0 & 2.321971 & -3.915812 & 1.672536 \\
\hline 45 & 1 & 0 & 3.254391 & -3.953304 & 1.895946 \\
\hline 46 & 1 & 0 & -5.000744 & -0.837572 & 2.083860 \\
\hline 47 & 1 & 0 & 0.549118 & 4.856221 & 2.215515 \\
\hline 48 & 1 & 0 & 1.847726 & -3.437680 & 2.356678 \\
\hline
\end{tabular}


49

8

0

0.220009

2.396814

2.650584

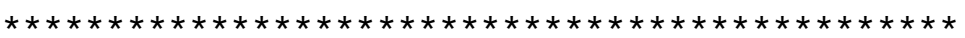

Gaussian 98: x86-Win32-G98RevA.11 25-Sep-2001

27-Jan-2003
\end{abstract}

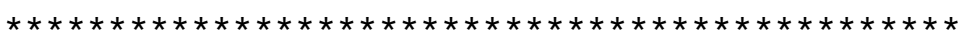

5a, DFT, 4C1 conformation of the galactose pentaacetate

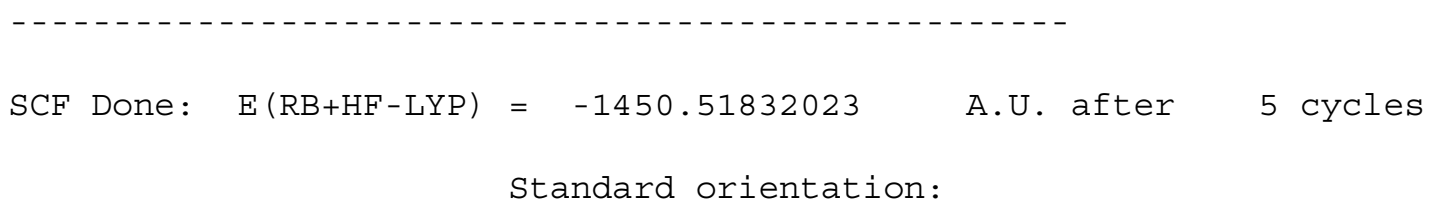

\begin{tabular}{|c|c|c|c|c|c|}
\hline Center & Atomic & Atomic & \multicolumn{3}{|c|}{ Coordinates (Angstroms) } \\
\hline Number & Number & Type & $\mathrm{X}$ & $\mathrm{Y}$ & $\mathrm{Z}$ \\
\hline 1 & 1 & 0 & -3.690142 & 0.753120 & -2.626723 \\
\hline 2 & 6 & 0 & -3.050365 & 1.586413 & -2.317981 \\
\hline 3 & 1 & 0 & -2.232987 & 1.675897 & -3.035994 \\
\hline 4 & 1 & 0 & -3.642929 & 2.500562 & -2.288195 \\
\hline 5 & 6 & 0 & -2.532509 & 1.278018 & -0.939782 \\
\hline 6 & 8 & 0 & -1.491313 & 0.402937 & -1.002919 \\
\hline 7 & 8 & 0 & -2.992254 & 1.702433 & 0.099232 \\
\hline 8 & 6 & 0 & -0.939194 & -0.069753 & 0.242651 \\
\hline 9 & 6 & 0 & -0.712261 & -1.578274 & 0.125652 \\
\hline 10 & 6 & 0 & 0.390165 & 0.648957 & 0.508554 \\
\hline 11 & 1 & 0 & -1.639646 & 0.147784 & 1.050248 \\
\hline 12 & 8 & 0 & 0.301809 & -1.887798 & -0.843227 \\
\hline 13 & 6 & 0 & -1.948717 & -2.340510 & -0.347554 \\
\hline
\end{tabular}




\begin{tabular}{|c|c|c|c|c|c|}
\hline 14 & 6 & 0 & 1.411080 & 0.259424 & -0.548026 \\
\hline 15 & 8 & 0 & 0.194963 & 2.068997 & 0.450110 \\
\hline 16 & 1 & 0 & -0.403485 & -1.946361 & 1.112516 \\
\hline 17 & 1 & 0 & 0.747358 & 0.375757 & 1.504336 \\
\hline 18 & 1 & 0 & -2.227872 & -2.031951 & -1.354356 \\
\hline 19 & 1 & 0 & 1.112582 & 0.629580 & -1.530643 \\
\hline 20 & 1 & 0 & -1.748572 & -3.414580 & -0.333709 \\
\hline 21 & 6 & 0 & 1.533934 & -1.275178 & -0.629383 \\
\hline 22 & 8 & 0 & 2.679033 & 0.818175 & -0.184514 \\
\hline 23 & 8 & 0 & -3.024312 & -2.072478 & 0.569764 \\
\hline 24 & 6 & 0 & -0.186673 & 2.672243 & 1.614005 \\
\hline 25 & 1 & 0 & 2.193585 & -1.565336 & -1.446908 \\
\hline 26 & 6 & 0 & 3.526594 & 1.122514 & -1.214139 \\
\hline 27 & 6 & 0 & -4.177093 & -1.557712 & 0.056871 \\
\hline 28 & 8 & 0 & 2.081460 & -1.769742 & 0.615822 \\
\hline 29 & 6 & 0 & -0.523933 & 4.119136 & 1.373769 \\
\hline 30 & 8 & 0 & -0.264379 & 2.090954 & 2.672029 \\
\hline 31 & 8 & 0 & 3.221747 & 1.021311 & -2.379221 \\
\hline 32 & 8 & 0 & -4.373059 & -1.384792 & -1.125860 \\
\hline 33 & 6 & 0 & 4.866166 & 1.554024 & -0.681685 \\
\hline 34 & 6 & 0 & 3.409332 & -2.067031 & 0.660355 \\
\hline 35 & 1 & 0 & 0.176331 & 4.576995 & 0.672088 \\
\hline 36 & 1 & 0 & -1.525066 & 4.162333 & 0.931970 \\
\hline 37 & 6 & 0 & -5.135333 & -1.194112 & 1.160149 \\
\hline 38 & 1 & 0 & -0.528264 & 4.654854 & 2.322641 \\
\hline 39 & 8 & 0 & 4.168020 & -1.915080 & -0.270330 \\
\hline 40 & 1 & 0 & 5.430023 & 2.048335 & -1.472166 \\
\hline 41 & 1 & 0 & 4.752868 & 2.211477 & 0.183048 \\
\hline
\end{tabular}




$\begin{array}{rrrrrr}42 & 1 & 0 & -4.902247 & -0.173205 & 1.482347 \\ 43 & 1 & 0 & 5.405315 & 0.657284 & -0.359167 \\ 44 & 1 & 0 & -6.155276 & -1.212456 & 0.775716 \\ 46 & 6 & 0 & 3.784091 & -2.596268 & 2.021371 \\ 47 & 1 & 0 & -5.030121 & -1.859601 & 2.018275 \\ 48 & 1 & 0 & 4.832215 & -2.892825 & 2.018682 \\ 49 & 1 & 0 & 3.620159 & -1.820552 & 2.775564 \\ & 1 & 0 & 3.150748 & -3.447786 & 2.284402\end{array}$

